Supplement of Geosci. Model Dev., 14, 1533-1551, 2021

https://doi.org/10.5194/gmd-14-1533-2021-supplement

(C) Author(s) 2021. CC BY 4.0 License.

(c) (i)

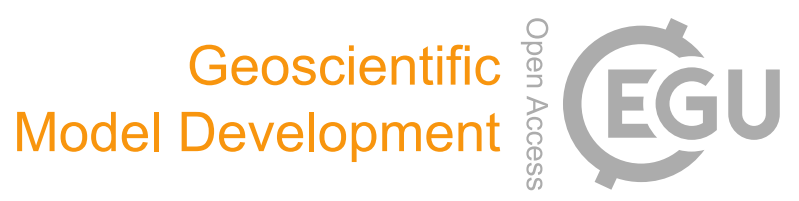

Supplement of

Sensitivity of surface solar radiation to aerosol-radiation and aerosol-cloud interactions over Europe in WRFv3.6.1 climatic runs with fully interactive aerosols

Sonia Jerez et al.

Correspondence to: Sonia Jerez (sonia.jerez@gmail.com)

The copyright of individual parts of the supplement might differ from the article licence. 


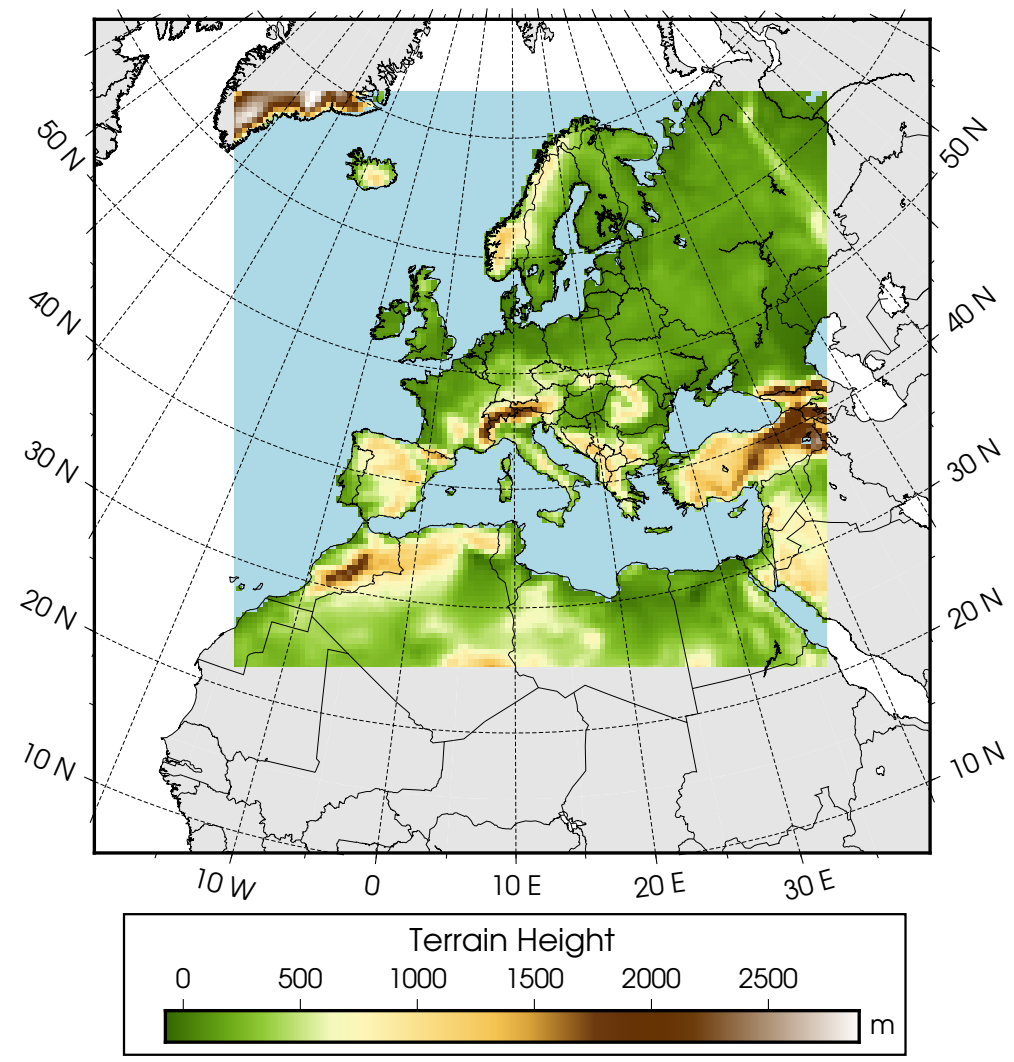

Supplementary Figure S1. Spatial domains used for the WRF simulations. The outer domain (gray shaded) has an horizontal resolution of $1.32^{\circ}$ in latitude and longitude; the inner target domain (colored) of $0.44^{\circ}$ (Euro-Cordex compliant). The nesting was one-way (outer-to-inner domain). 
RSDS JJA climatologies for 1991-2010

(a) GCM

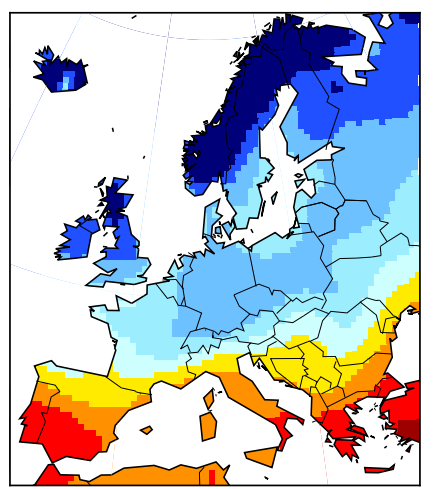

(d) BASE

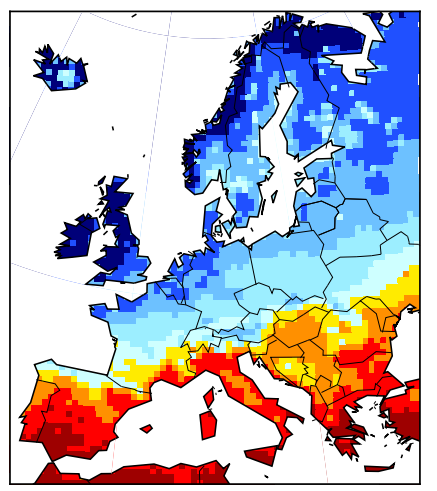

(g) BASE-GCM

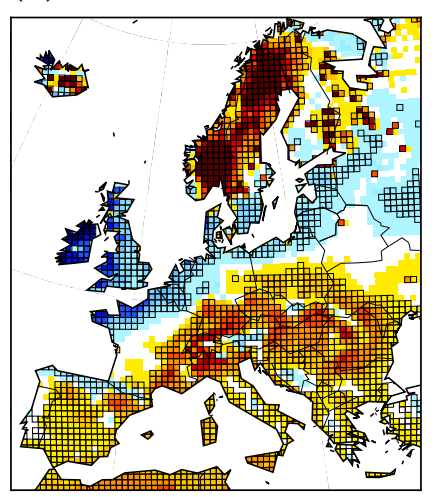

(j) BASE-ERA5

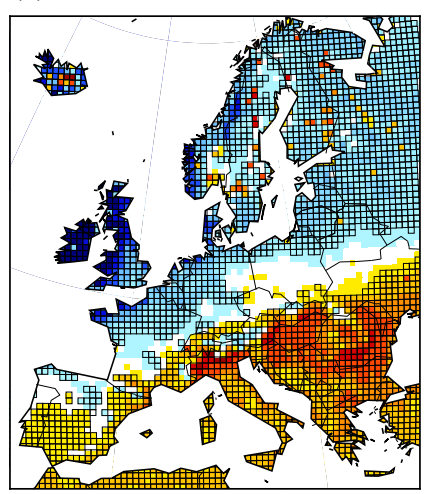

(b) ERA5

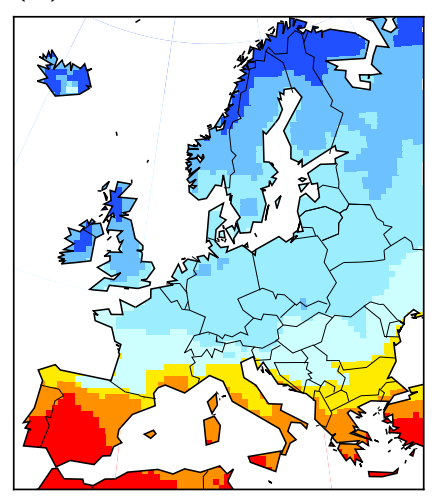

(e) ARI

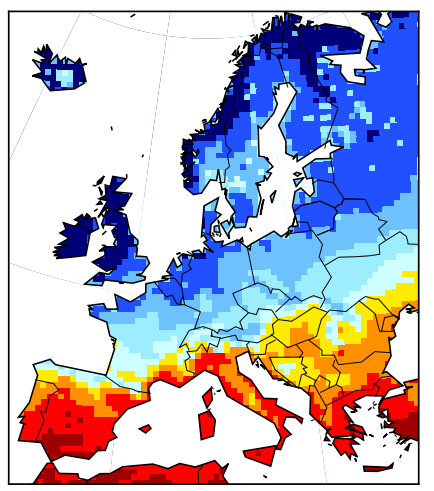

(h) ARI-GCM

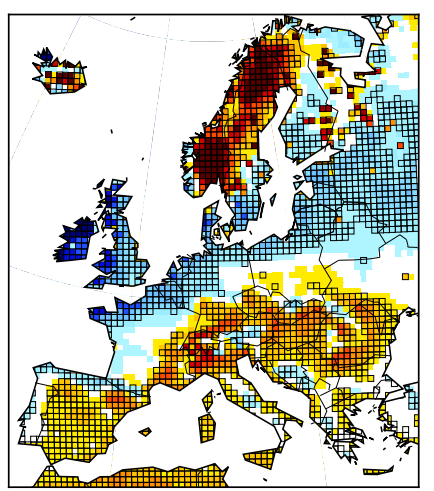

(k) ARI-ERA5

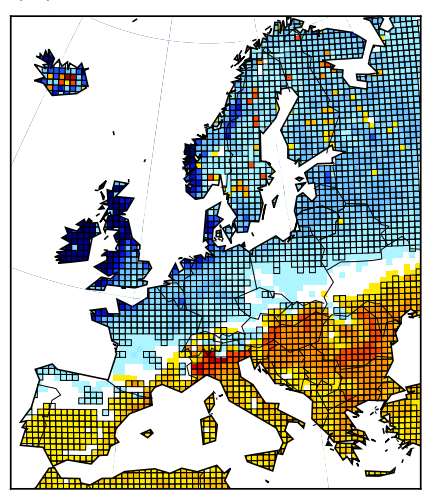

(c) GCM-ERA5

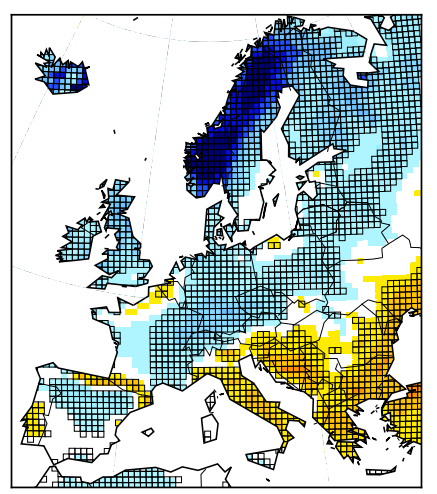

(f) ARCI

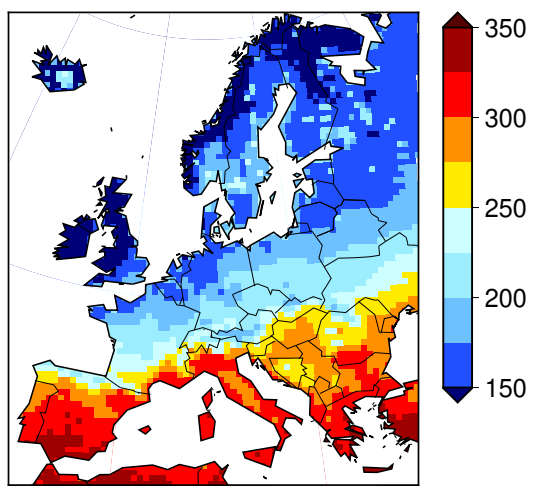

(i) ARCI-GCM

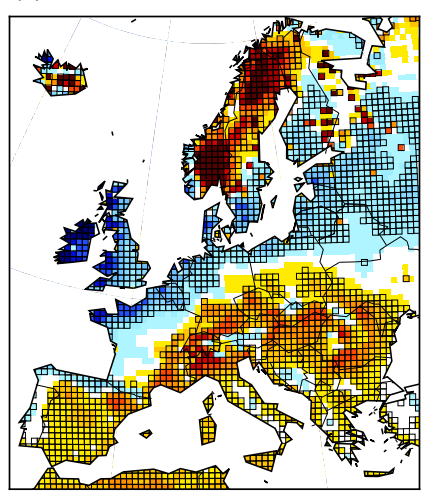

(l) ARCI-ERA5

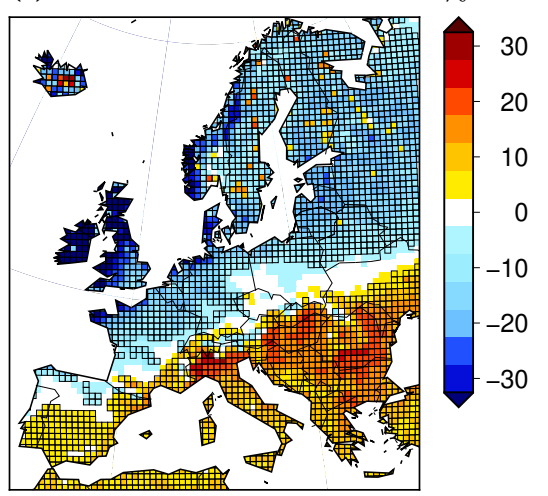

Supplementary Figure S2. RSDS summer climatologies in the historical period from the GCM (a), ERA5 (b) and the WRF simulations ( $\mathrm{d}$ to f); units: $\mathrm{Wm}^{-2}$, same colorbar in all cases (the upper one). Panel c depicts relative differences between the GCM and ERA5, panels g to i between each WRF simulation and the GCM, and panels $\mathrm{j}$ to $\mathrm{l}$ between each WRF simulation and ERA5, squared if statistically significant $(\mathrm{p}<0.05)$; units: \%, same colorbar in all cases (the bottom one). 
RSDS, CCT \& AOD JJA climatologies for 1991-2010: differences between experiments

(a) RSDS ARI-BASE

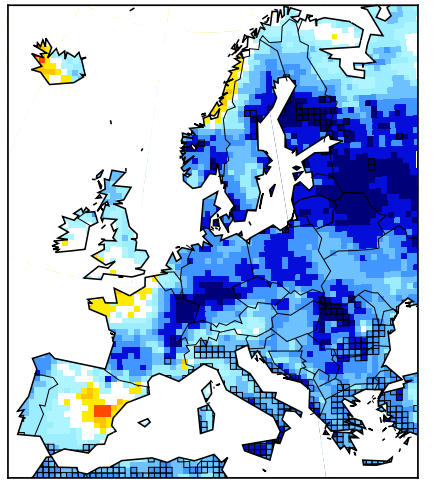

(d) CCT ARI-BASE

s_corr with (a) $=-0.75$

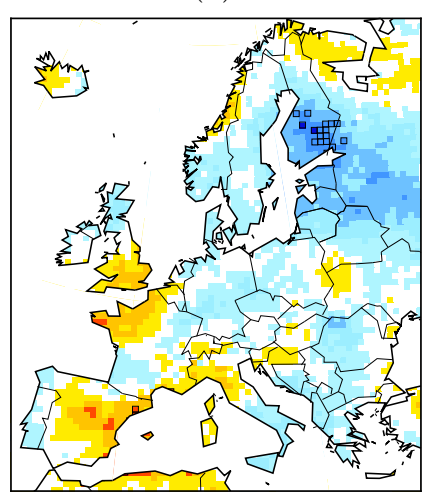

(g) AOD ARI

s_corr with (a) $=-0.15$

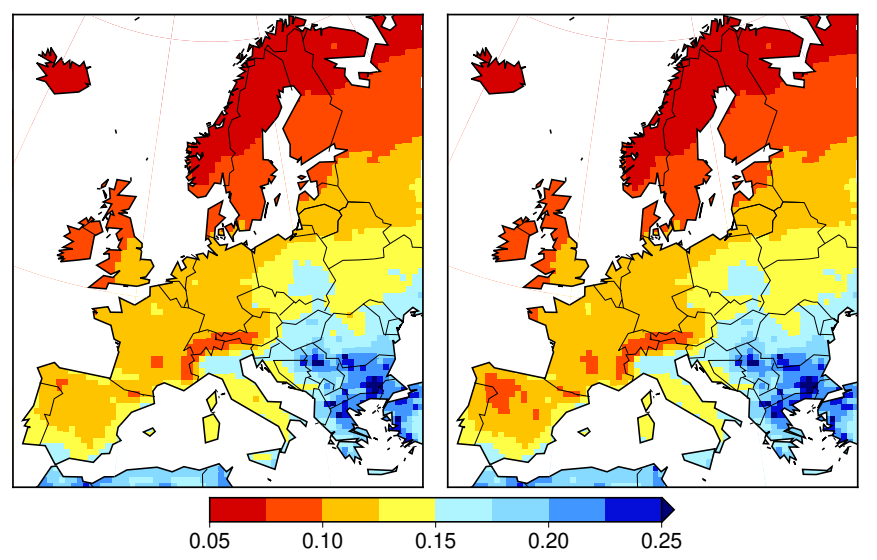

(c) RSDS ARCI-ARI

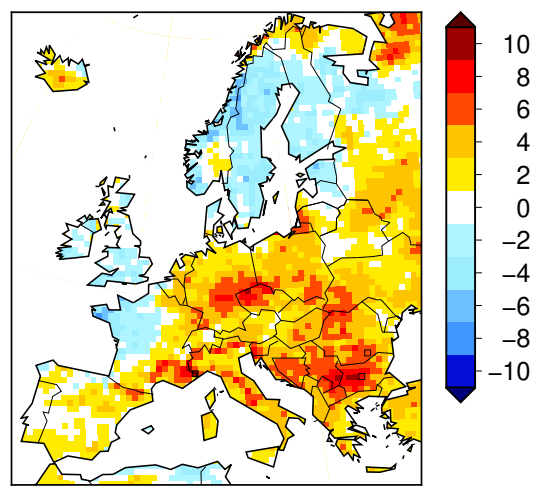

(f) CCT ARCI-ARI

$s_{-}$corr with $(\mathrm{c})=-0.65$

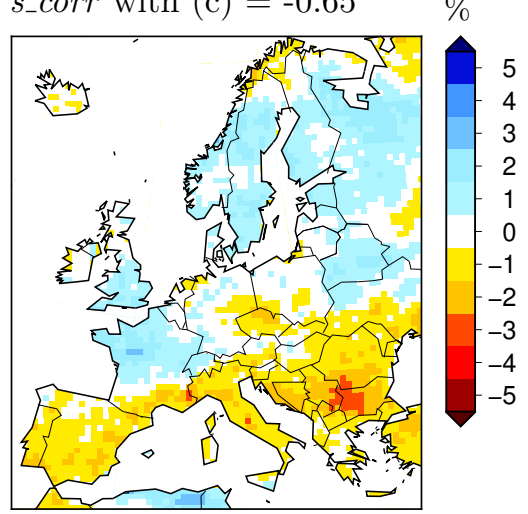

(i) AOD ARCI-ARI

$s_{-}$corr with $(\mathrm{c})=-0.05$

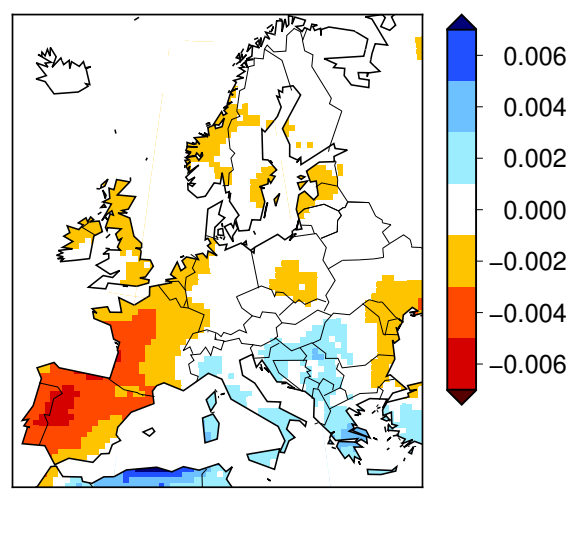

Supplementary Figure S3. As Figure 1 of the main manuscript but with plain differences depicted (non-relative values). Units: RSDS in $\mathrm{Wm}^{-2}$, CCT in \% of the grid cell area, AOD dimensionless. 
Temporal correlations between RSDS, CCT \& AOD differences JJA-mean series in 1991-2010

(a) RSDS vs CTT diffs ARI-BASE

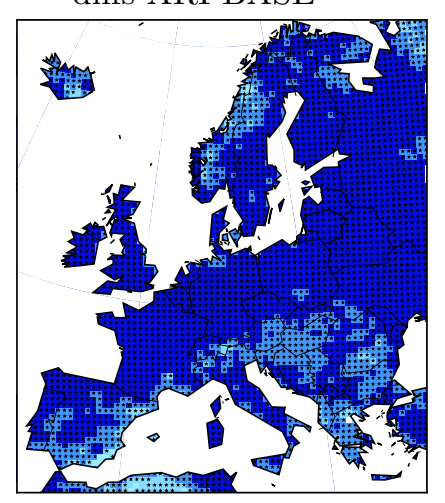

(d) RSDS vs AOD diffs ARI-BASE

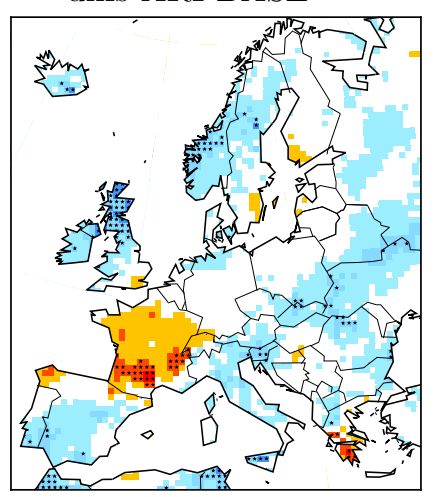

(g) $\mathrm{RSDS}_{c s}$ vs $\mathrm{AOD}_{c s}$ diffs ARI-BASE

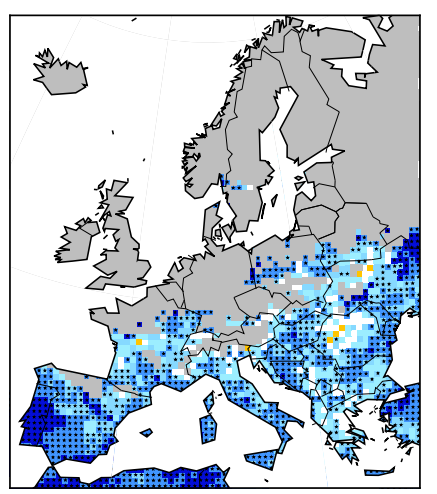

(b) RSDS vs CTT diffs ARCI-BASE

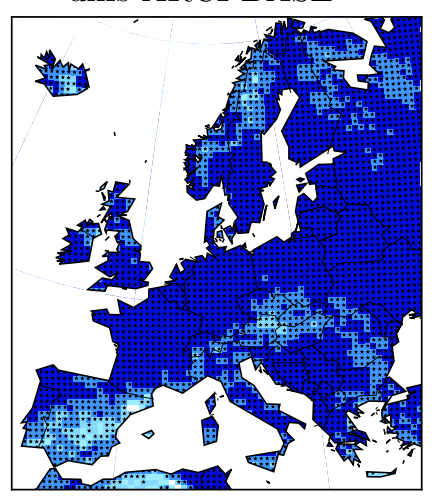

(e) RSDS vs AOD diffs ARCI-BASE

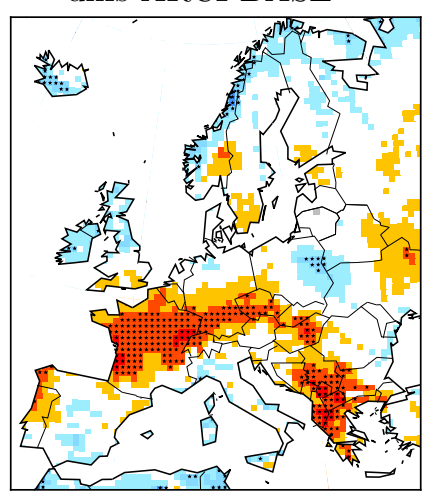

(h) $\mathrm{RSDS}_{c s}$ vs $\mathrm{AOD}_{c s}$ diffs ARCI-BASE

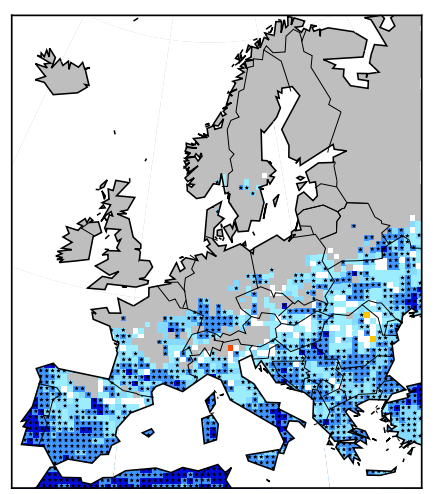

(c) RSDS vs CTT diffs ARCI-ARI

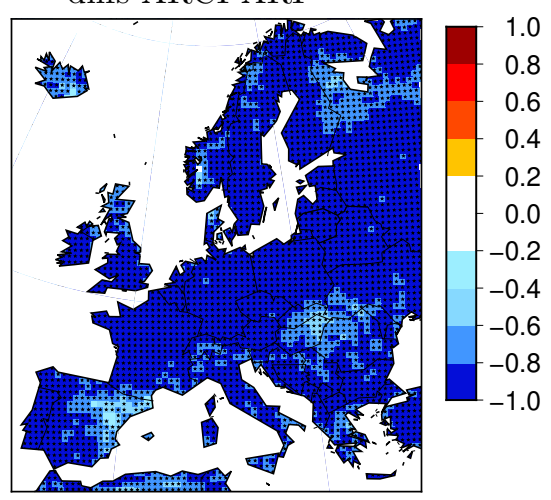

(f) RSDS vs AOD diffs ARCI-ARI

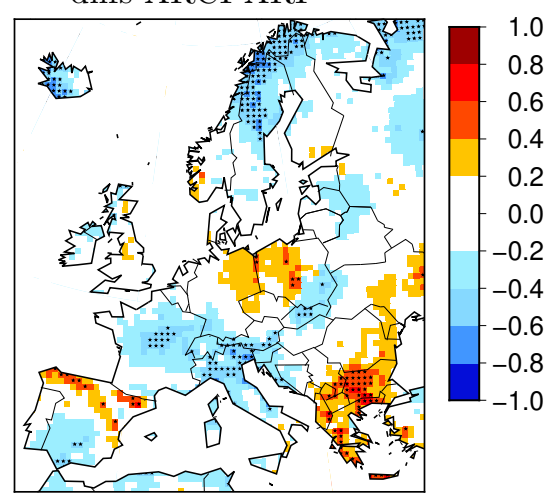

(i) $\mathrm{RSDS}_{c s}$ vs $\mathrm{AOD}_{c s}$ diffs ARCI-ARI

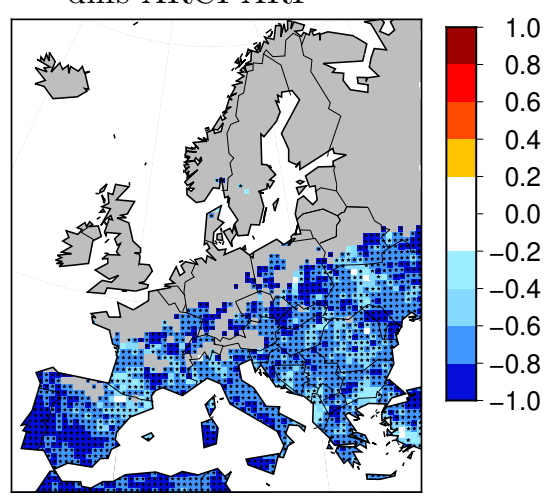

Supplementary Figure S4. Temporal correlations between JJA-mean temporal series of differences in RSDS and CTT (a to c), RSDS and AOD (d to f), and $\mathrm{RSDS}_{c s}$ and $\mathrm{AOD}_{c s}$ (g to i; gray-shaded areas where the number of time steps in the clear-sky series is below $75 \%$ of total time steps) between ARI and BASE (first column), ARCI and BASE (second column), and ARCI and ARI experiments (third column) in the historical period (1991-2010). Little stars indicate statistical significance $(\mathrm{p}<0.05)$. 
$\mathrm{RSDS}_{c s} \& \mathrm{AOD}_{c s}$ JJA climatologies for 1991-2010: differences between experiments
(a) $\operatorname{RSDS}_{c s}$ ARI-BASE

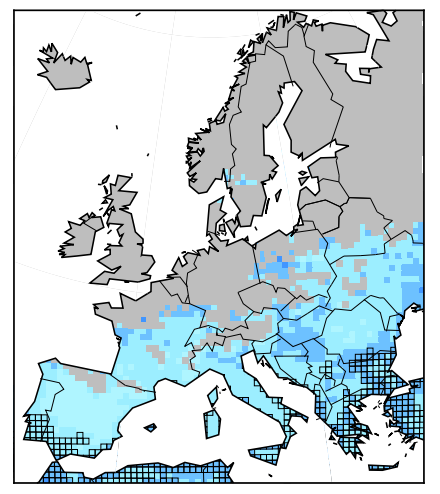

(d) $\mathrm{AOD}_{c s} \mathrm{ARI}$

$s_{-}$corr with $(\mathrm{a})=-0.66$

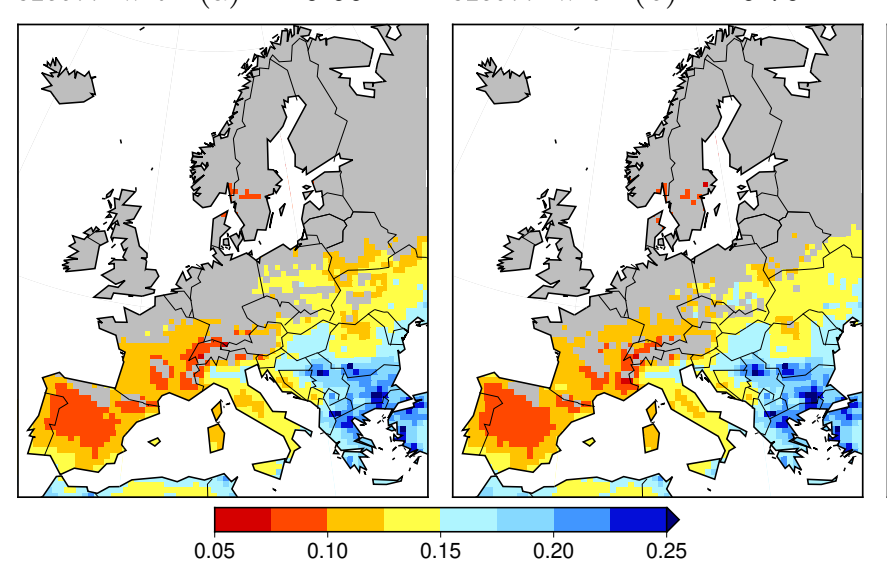

(c) $\operatorname{RSDS}_{c s}$ ARCI-ARI

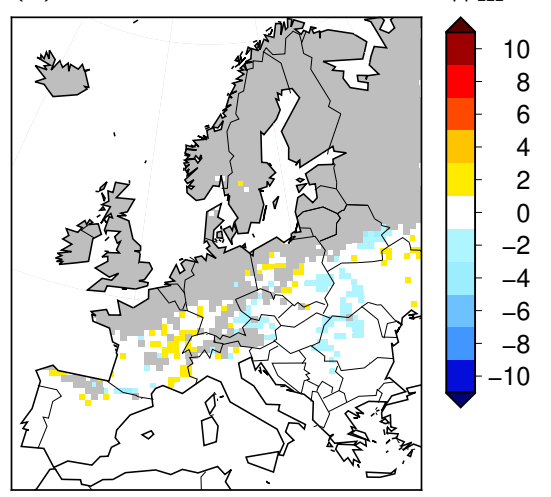

(f) $\mathrm{AOD}_{c s} \mathrm{ARCI}-\mathrm{ARI}$ $s_{-}$corr with $(\mathrm{c})=-0.67$

Supplementary Figure S5. As Figure 6 of the main manuscript but with plain differences depicted (non-relative values). Units: $\operatorname{RSDS}_{c s}$ in $\mathrm{Wm}^{-2}, \mathrm{AOD}_{c s}$ dimensionless.

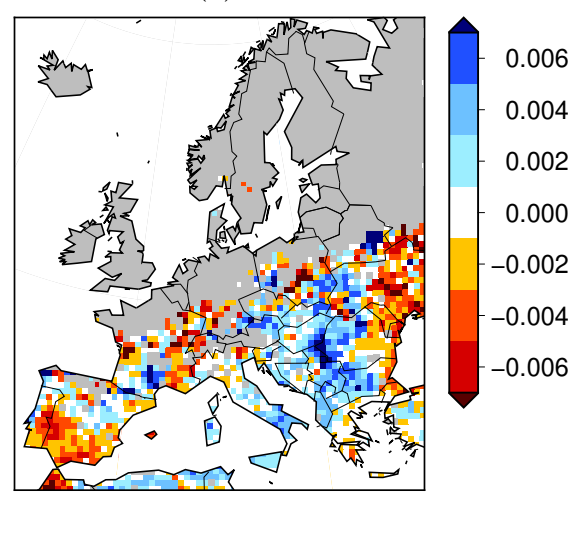


RSDS, CCT \& AOD JJA climatologies for 2031-2050: differences between experiments

(a) RSDS ARI-BASE

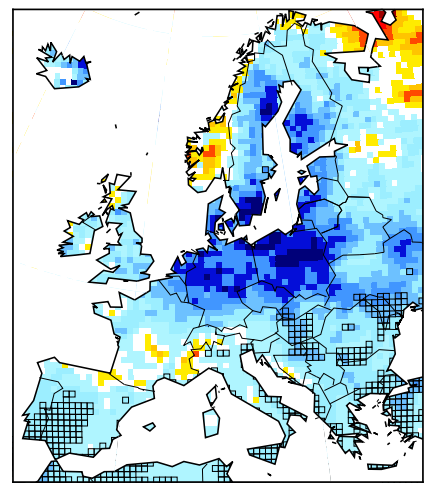

(d) CCT ARI-BASE

$s_{-}$corr with $(\mathrm{a})=-0.59$

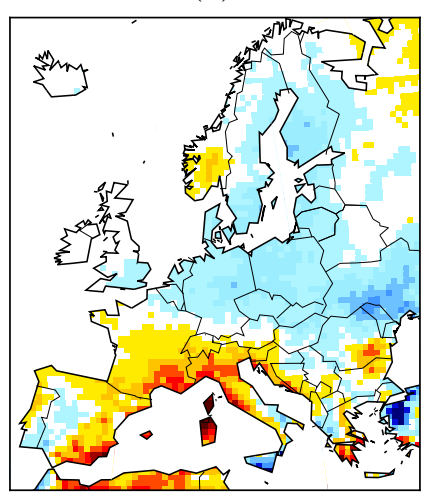

(g) AOD ARI

$s_{-}$corr with $(\mathrm{a})=-0.50$

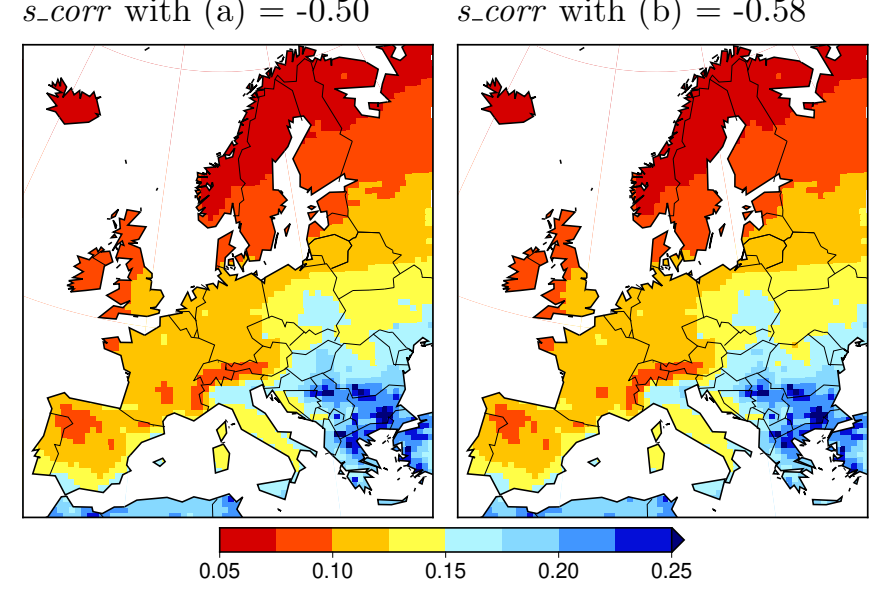

(c) RSDS ARCI-ARI

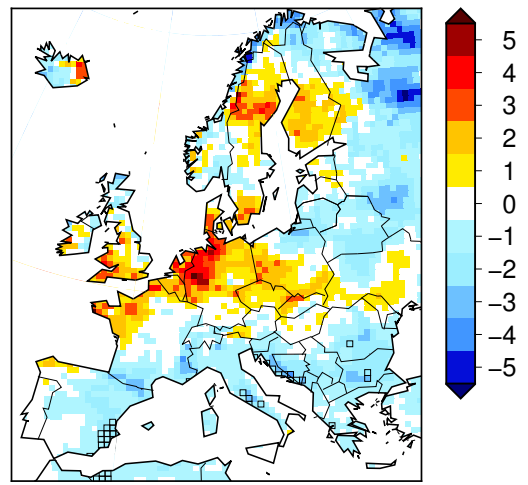

(f) CCT ARCI-ARI

$s_{-}$corr with $(\mathrm{c})=-0.74$

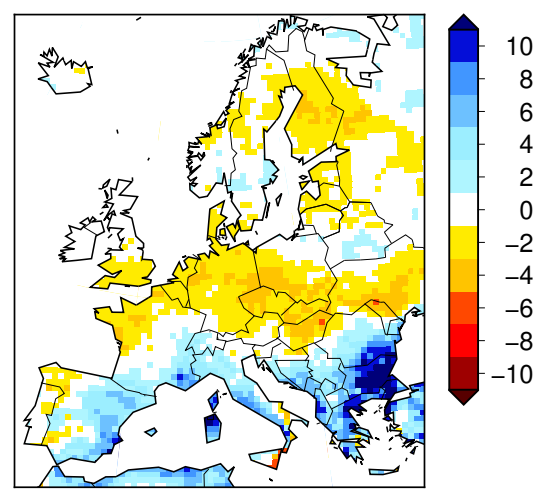

(i) AOD ARCI-ARI

$s_{-}$corr with (c) $=-0.12 \quad \%$

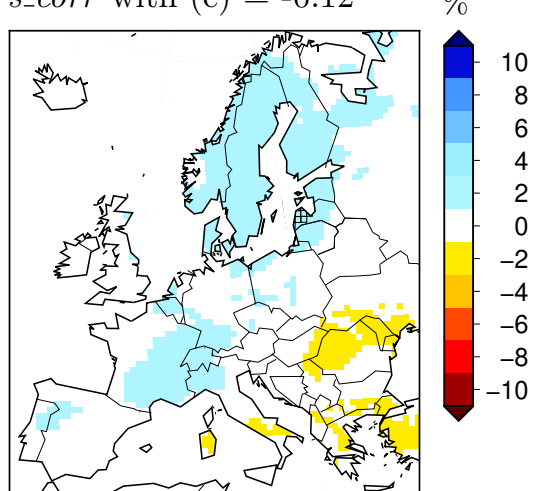

Supplementary Figure S6. As Figure 1 of the main manuscript but for the future period (2031-2050). 
Temporal correlations between RSDS, CCT \& AOD differences JJA-mean series in 2031-2050

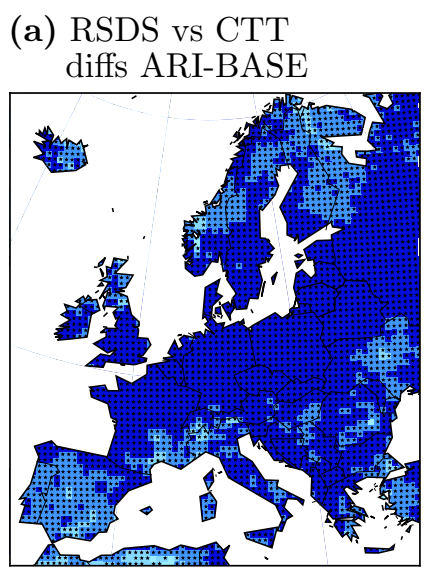

(d) RSDS vs AOD diffs ARI-BASE

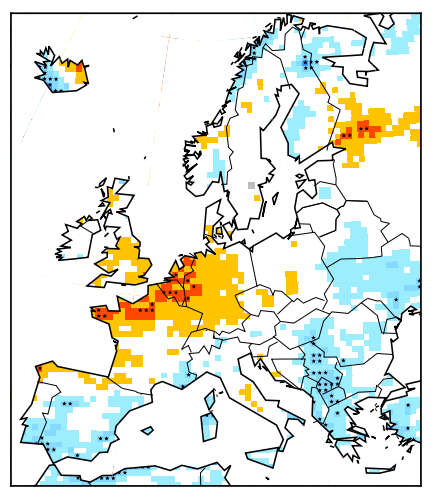

(g) $\mathrm{RSDS}_{c s}$ vs $\mathrm{AOD}_{c s}$ diffs btw ARI-BASE

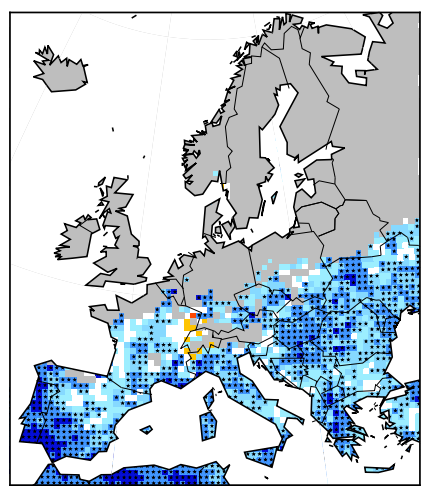

(b) RSDS vs CTT diffs ARCI-BASE

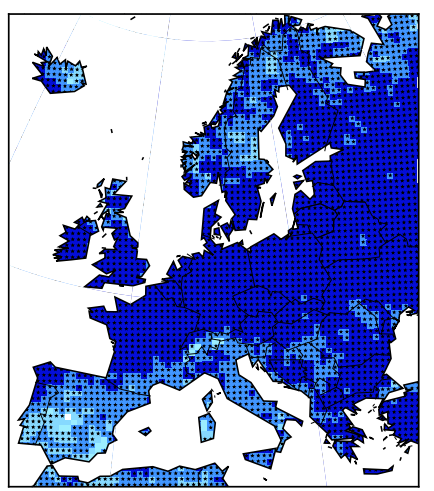

(e) RSDS vs AOD diffs ARCI-BASE

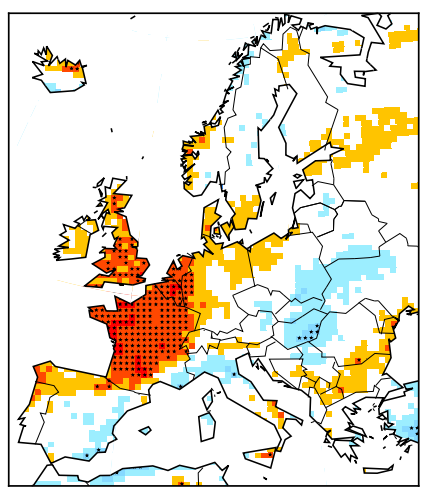

(h) $\mathrm{RSDS}_{c s}$ vs $\mathrm{AOD}_{c s}$ diffs ARCI-BASE

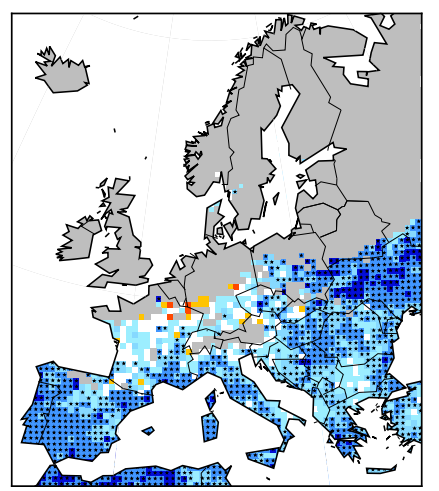

(c) RSDS vs CTT diffs ARCI-ARI

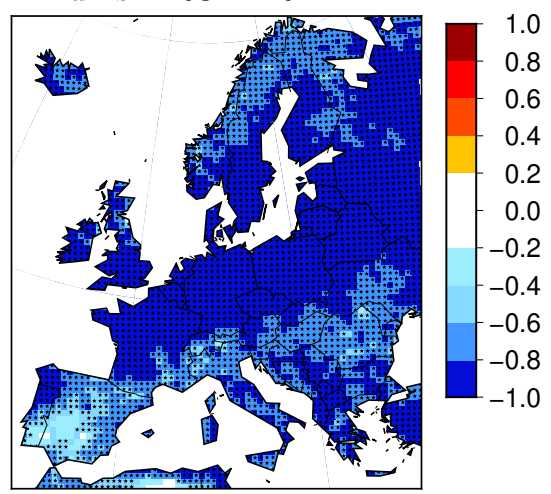

(f) RSDS vs AOD diffs ARCI-ARI

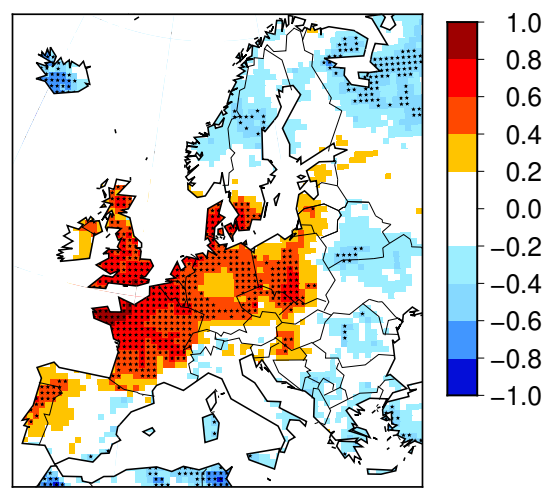

(i) $\operatorname{RSDS}_{c s}$ vs $\mathrm{AOD}_{c s}$ diffs ARCI \& ARI

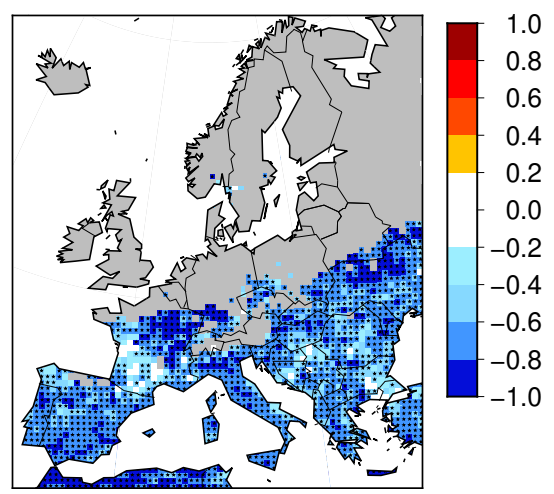

Supplementary Figure S7. As Supplementary Figure 4 but for the future period (2031-2050). 
$\mathrm{RSDS}_{c s} \& \mathrm{AOD}_{c s}$ JJA climatologies for 2031-2050: differences between experiments

\section{(a) $\operatorname{RSDS}_{c s}$ ARI-BASE}

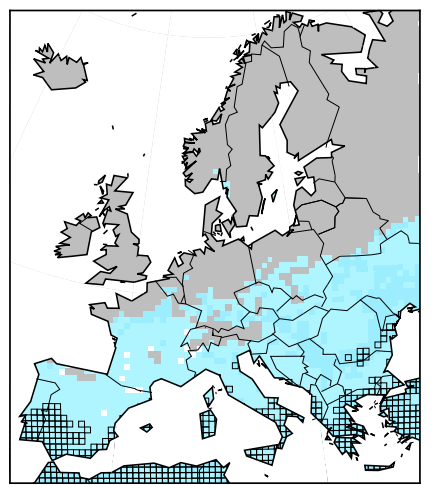

(d) $\mathrm{AOD}_{c s} \mathrm{ARI}$

s_corr with $(\mathrm{a})=-0.77$
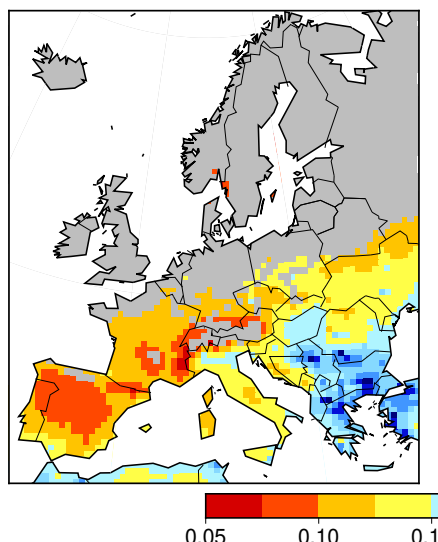

(b) $\mathrm{RSDS}_{c s}$ ARCI-BASE

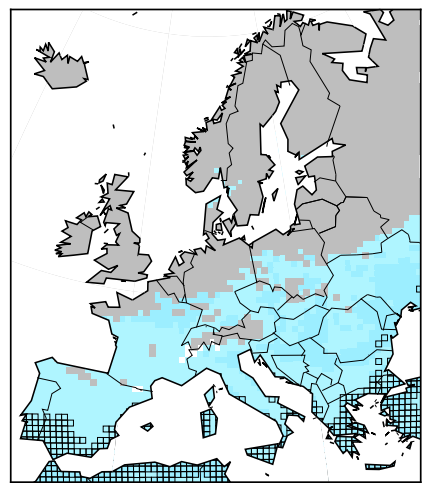

(e) $\mathrm{AOD}_{c s} \mathrm{ARCI}$

$s_{-}$corr with $(\mathrm{b})=-0.80$

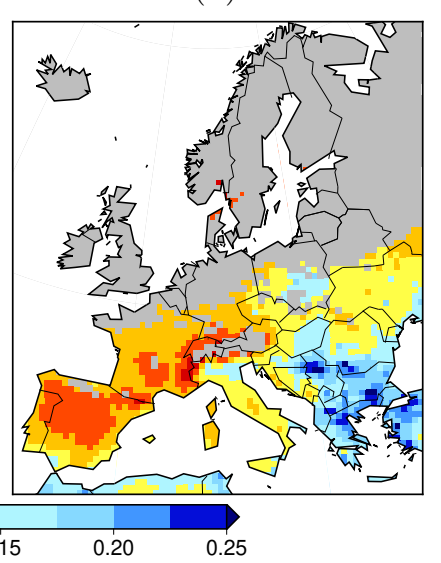

(c) $\operatorname{RSDS}_{c s}$ ARCI-ARI

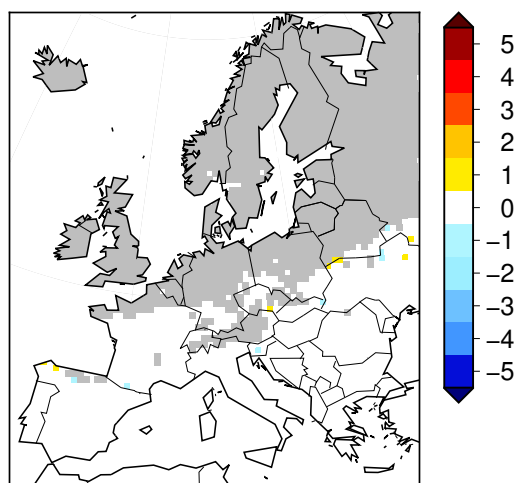

(f) $\mathrm{AOD}_{c s}$ ARCI-ARI $s_{-}$corr with $(\mathrm{c})=-0.61$

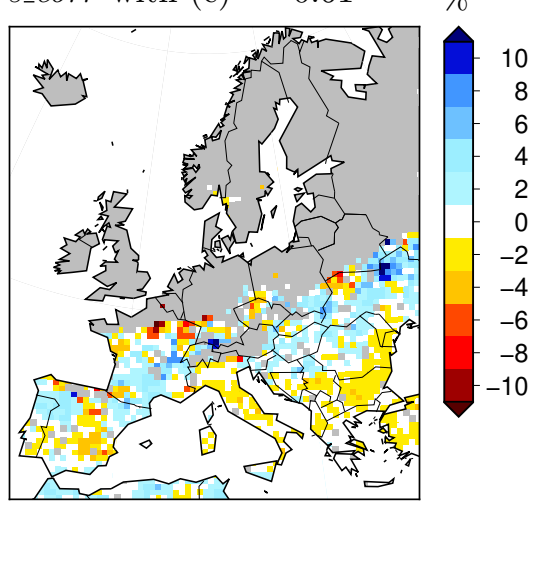

Supplementary Figure S8. As Figure 6 of the main manuscript but for the future period (2031-2050). 
RSOT, TAS, RH \& CLD JJA climatologies for 2031-2050: differences between experiments

(a) RSOT ARI-BASE

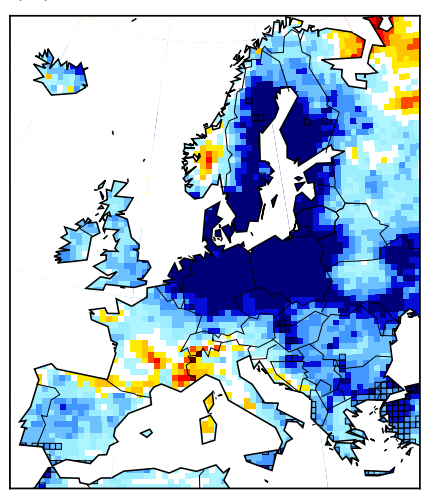

(d) TAS ARI-BASE

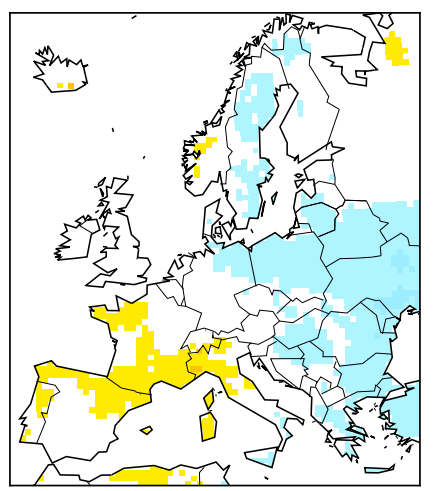

(g) RH ARI-BASE

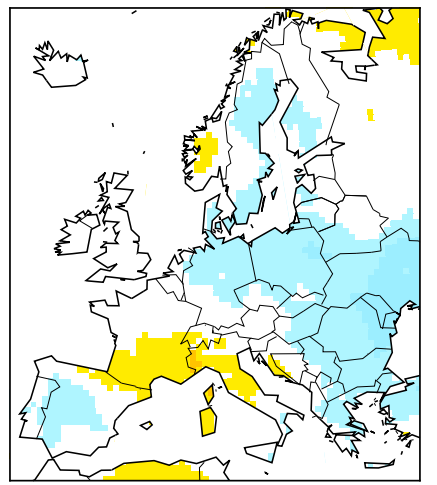

(j) CLD ARI-BASE

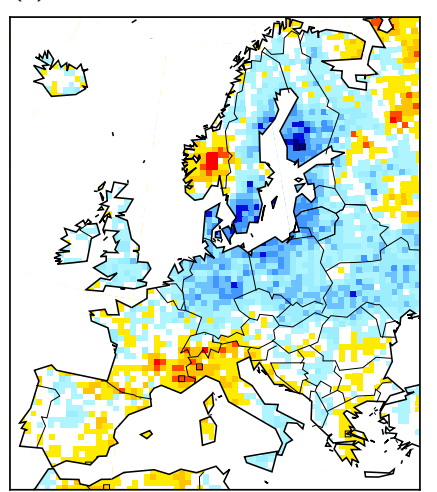

(b) RSOT ARCI-BASE

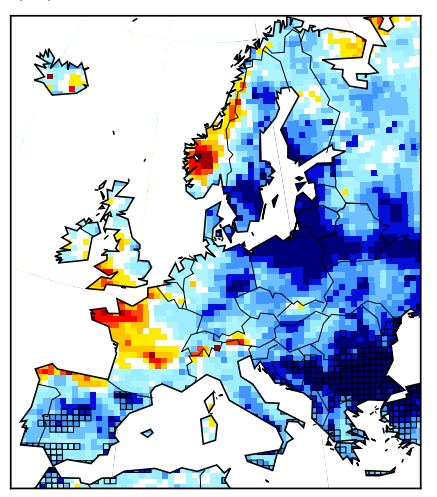

(e) TAS ARCI-BASE

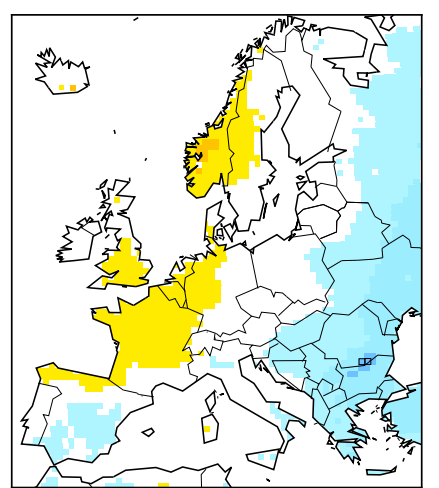

(h) RH ARCI-BASE

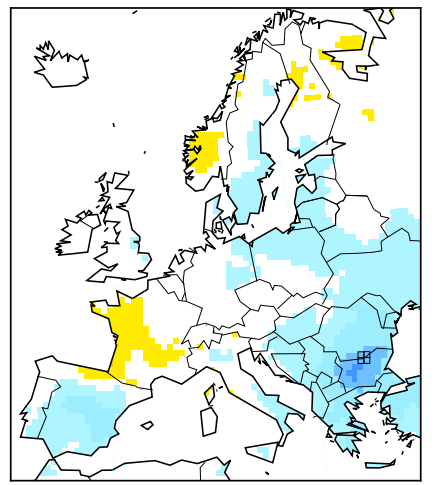

(k) CLD ARCI-BASE

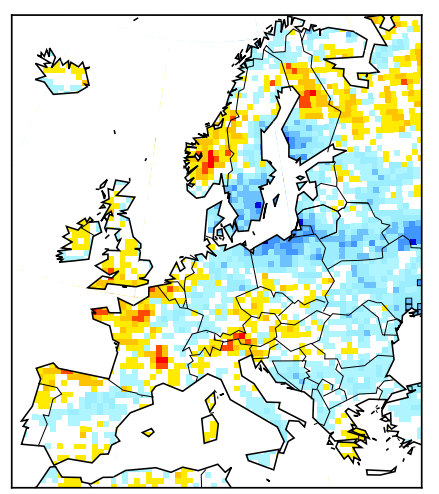

(c) RSOT ARCI-ARI

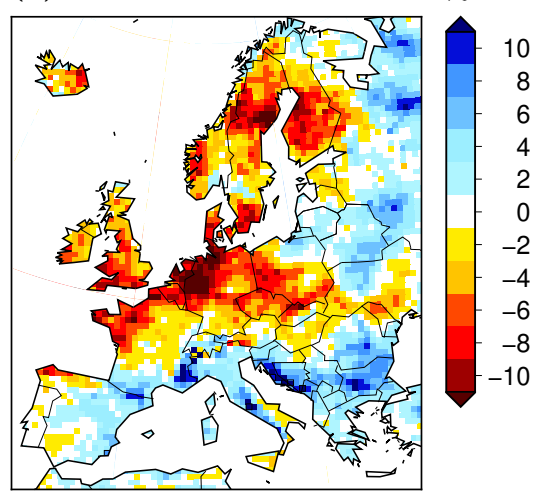

(f) TAS ARCI-ARI

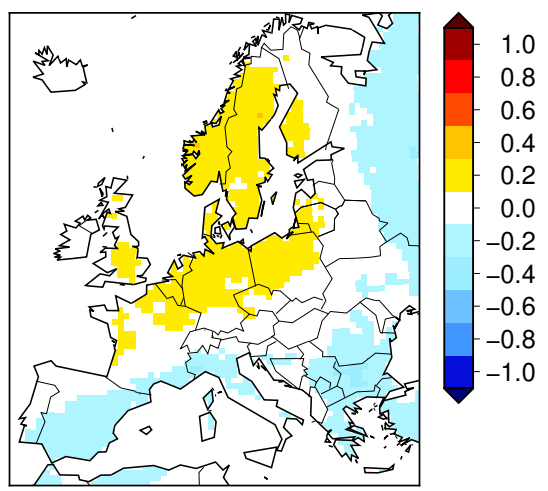

(i) RH ARCI-ARI

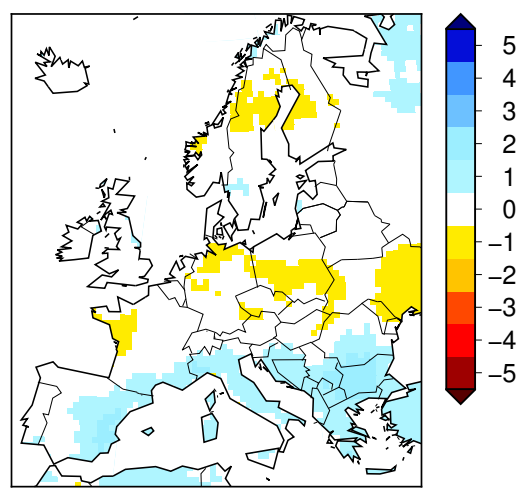

(l) CLD ARCI-ARI

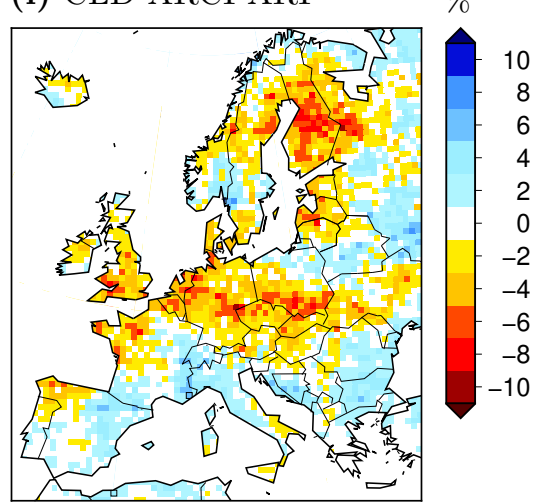

Supplementary Figure S9. As Figure 3 of the main manuscript but for the future period (2031-2050). 
Precipitation-related JJA climatologies for 2031-2050: differences between experiments

(a) PR ARI-BASE

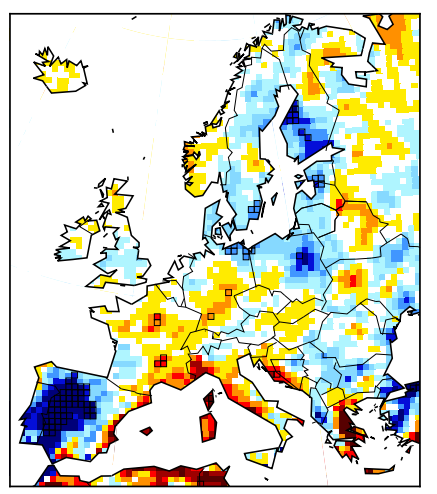

(d) 90pctl PR ARI-BASE

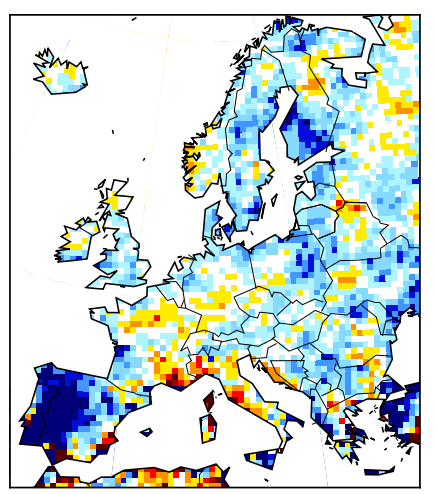

(g) RD ARI-BASE

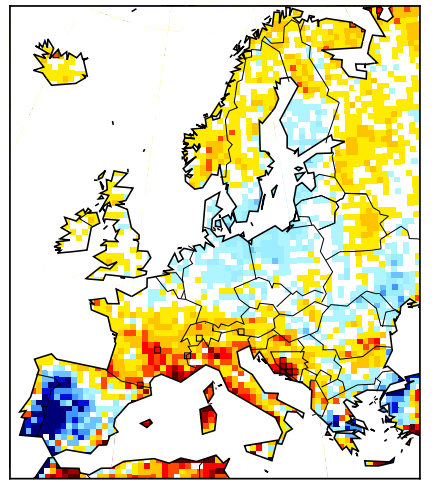

(j) PRC ARI-BASE

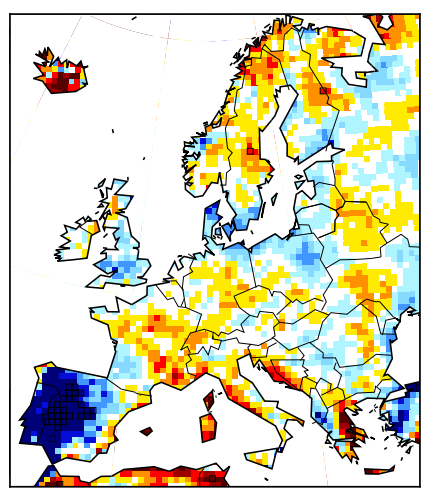

(b) PR ARCI-BASE

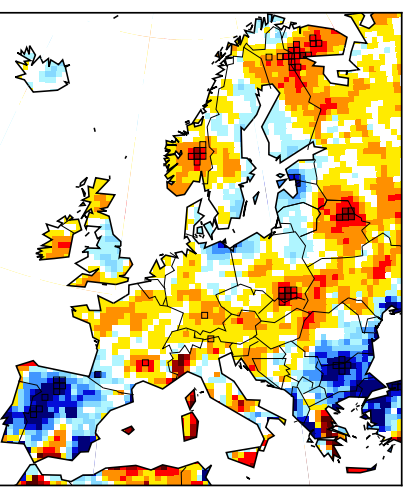

(e) 90pctl PR ARCI-BASE

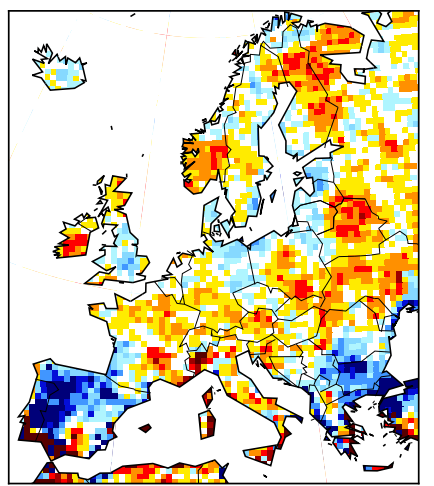

(h) RD ARCI-BASE

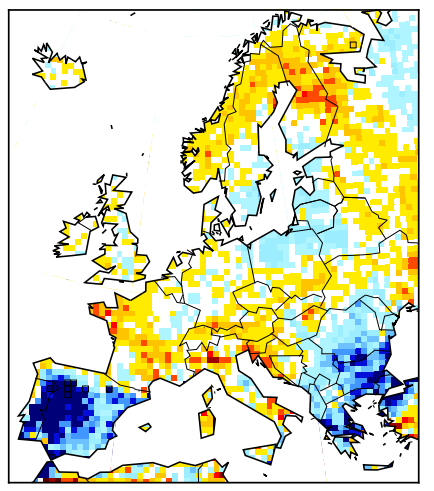

(k) PRC ARCI-BASE

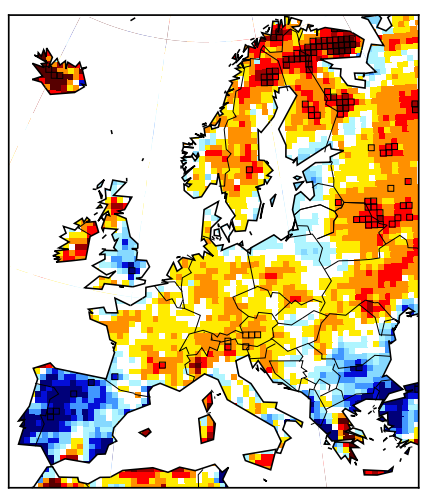

(c) PR ARCI-ARI

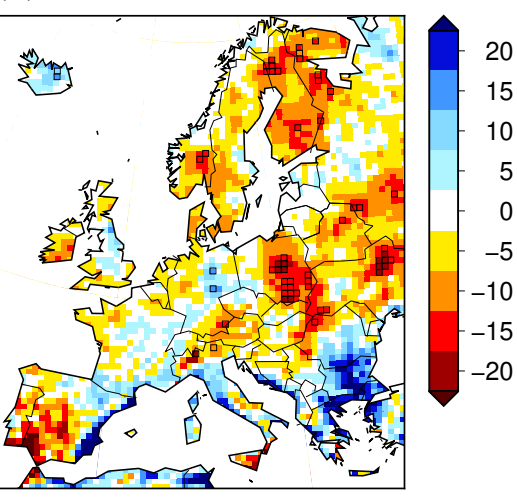

(f) 90pctl PR ARCI-ARI \%

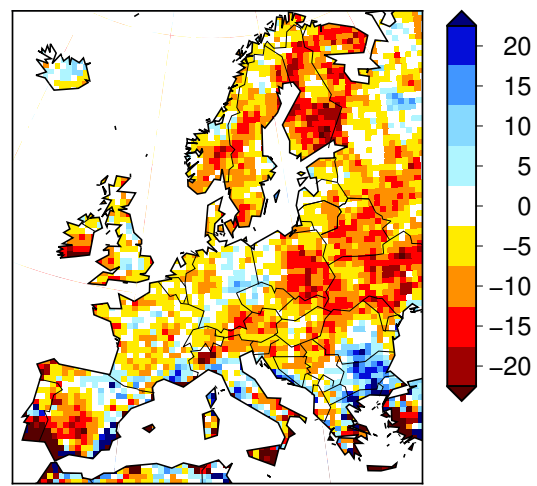

(i) RD ARCI-ARI

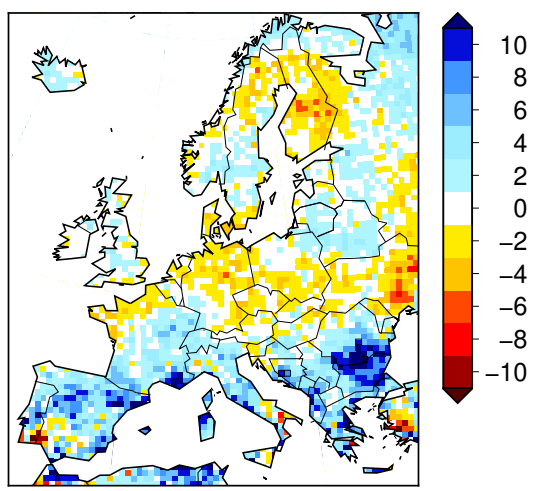

(l) PRC ARCI-ARI

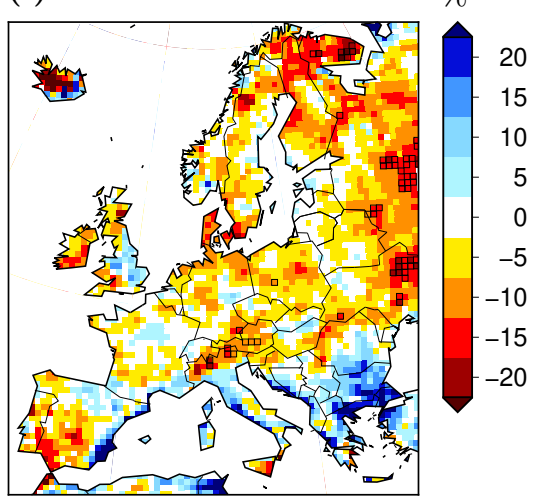

Supplementary Figure S10. As Figure 4 of the main manuscript but for the future period (2031-2050). 
Contribution of each aerosol species to the JJA-mean total surface aerosol concentration (period 2031-2050)

(k) ARI BC

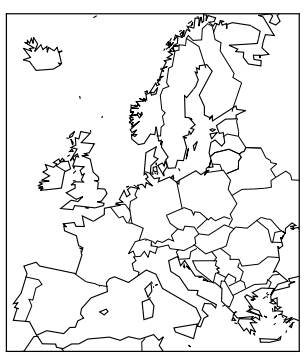

(p) ARCI BC
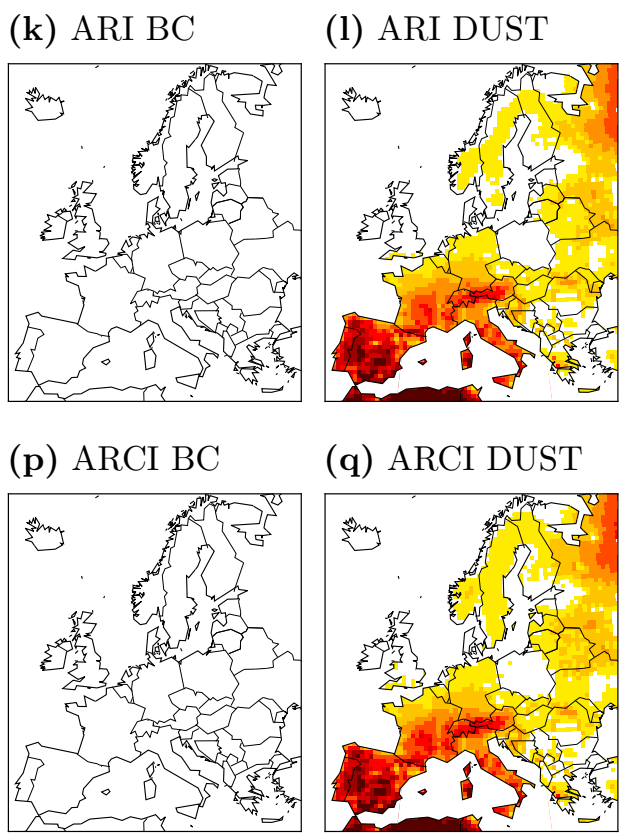

(q) ARCI DUST

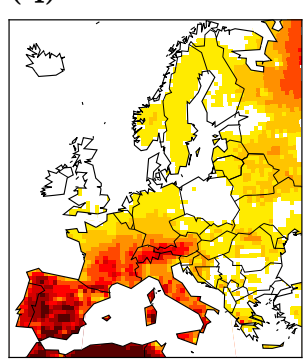

(m) ARI OC

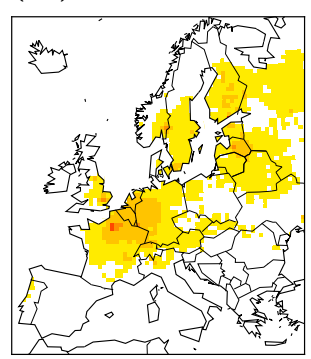

(n) ARI SEAS

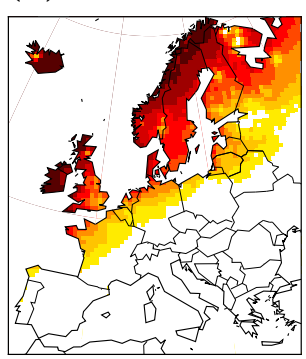

(r) ARCI OC

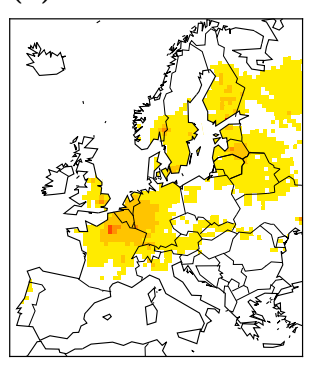

(s) ARCI SEAS

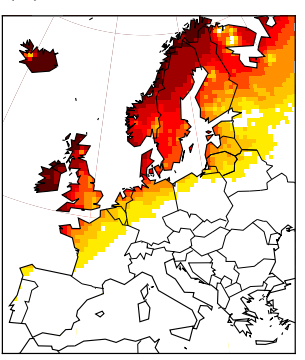

(o) ARI SULF

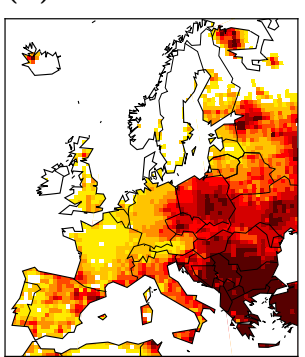

(t) ARCI SULF

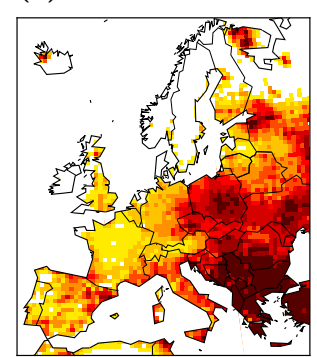

Ner

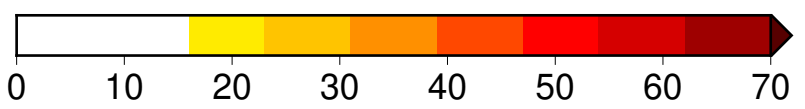

Supplementary Figure S11. As Figure 2 of the main manuscript but for the future period (2031-2050). 
RSDS, CCT \& AOD JJA changes (2031-2050 vs. 1991-2010)

(a) GCM RSDS

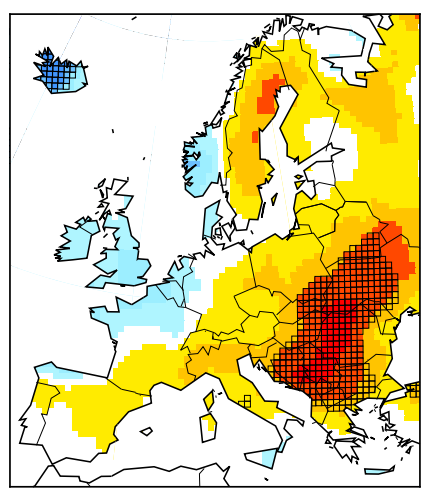

(e) GCM CCT

s_corr with (a) $=-0.91$

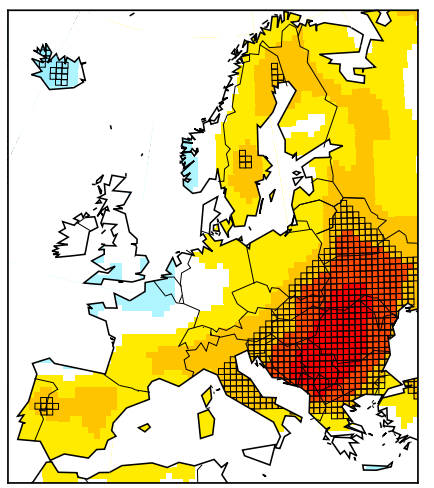

(i) GCM AOD

$s_{-} \operatorname{corr}$ with $(\mathrm{a})=-0.57$

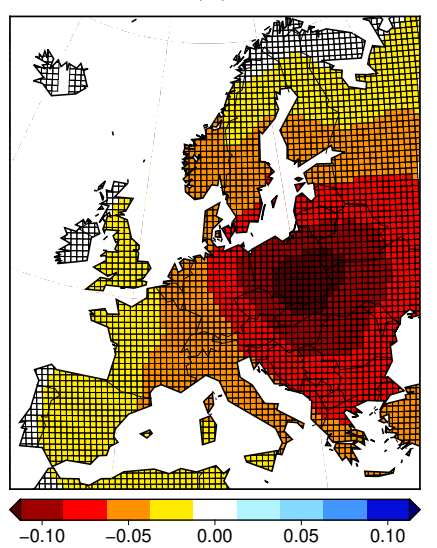

(b) BASE RSDS

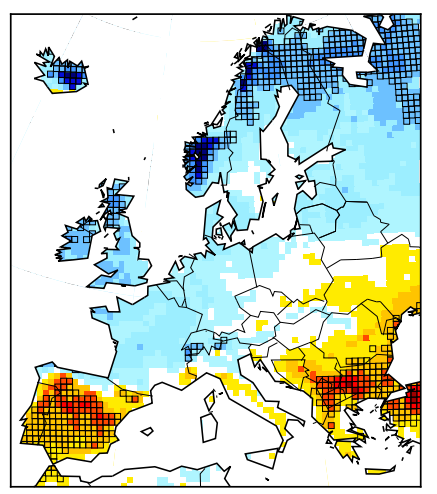

(f) BASE CCT

$s_{-}$corr with $(\mathrm{b})=-0.88$

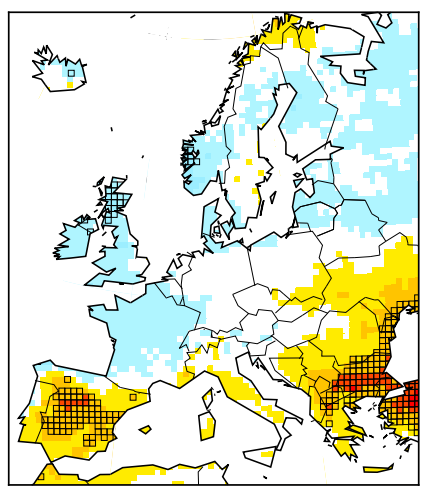

(j) BASE AOD

$s_{-}$corr with (b) = N.A.

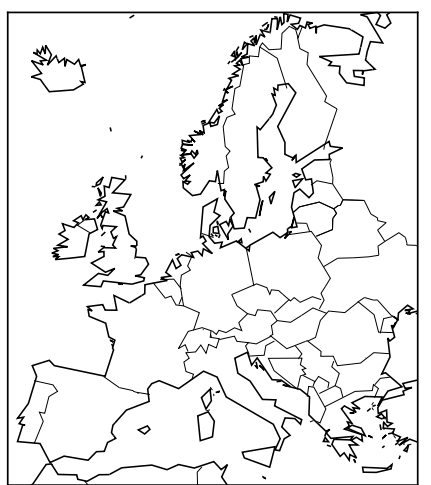

(c) ARI RSDS

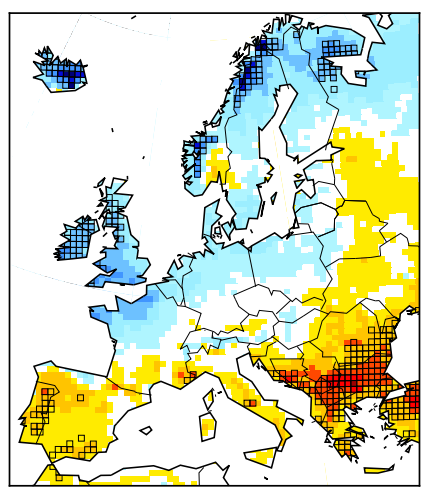

(g) ARI CCT

$s_{-}$corr with $(\mathrm{c})=-0.83$

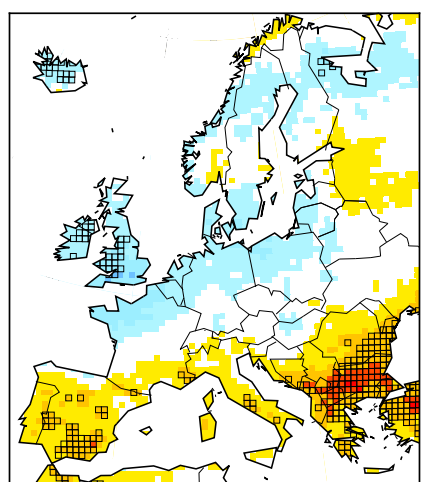

(k) ARI AOD

$s_{-}$corr with $(\mathrm{c})=0.21$

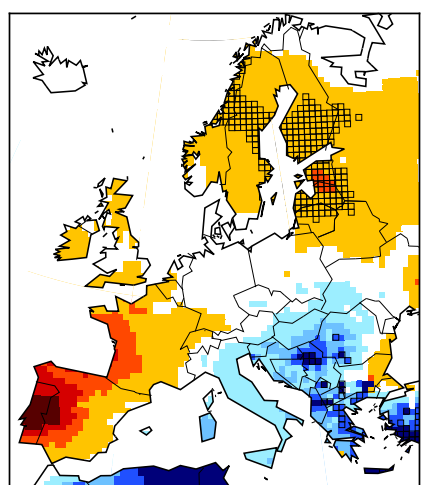

(d) ARCI RSDS

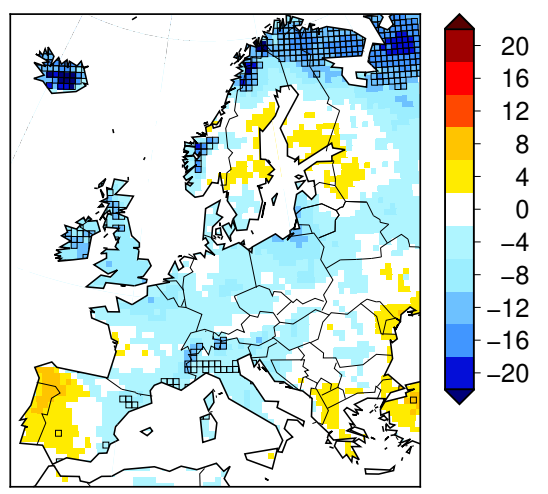

(h) ARCI CCT

$s_{-}$corr with $(\mathrm{d})=-0.77$

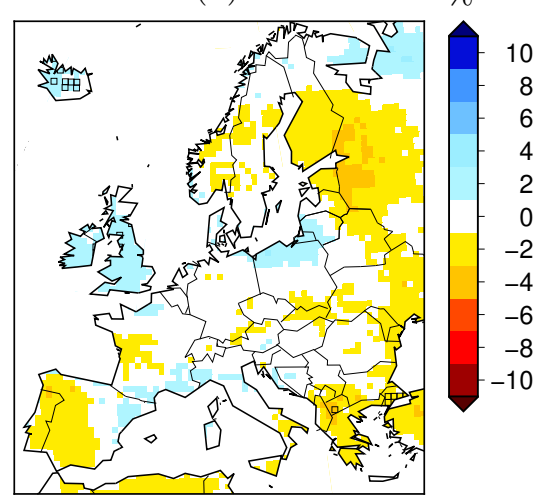

(l) ARCI AOD

s_corr with $(\mathrm{d})=-0.11$

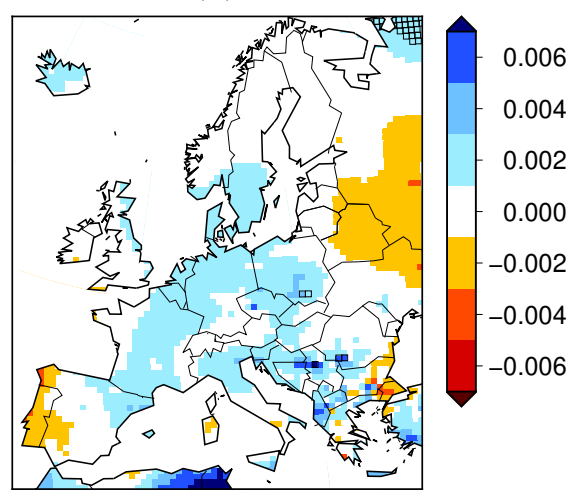

Supplementary Figure S12. As Figure 7 of the main manuscript but with plain differences depicted (non-relative values). Units: RSDS in $\mathrm{Wm}^{-2}$, CCT in \% of the grid cell area, AOD dimensionless. 
$\operatorname{RSDS}_{c s} \& \mathrm{AOD}_{c s}$ JJA changes (2031-2050 vs. 1991-2010)

(a) $\mathrm{BASE} \mathrm{RSDS}_{c s}$

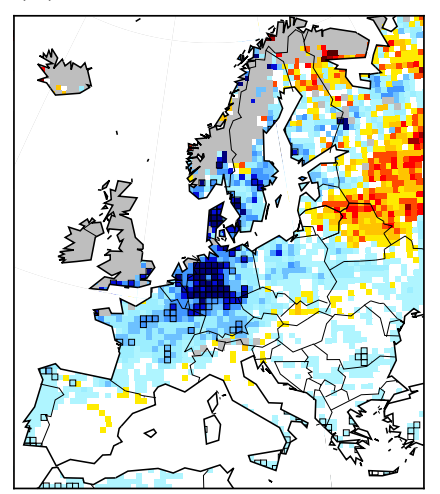

(d) BASE $\mathrm{AOD}_{c s}$

$s_{-}$corr with $(\mathrm{a})=$ N.A.

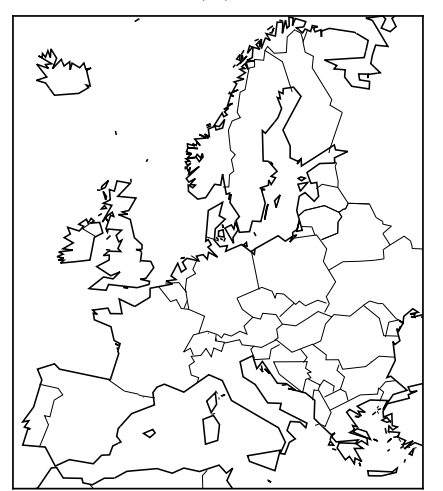

(b) ARI RSDS $c s$

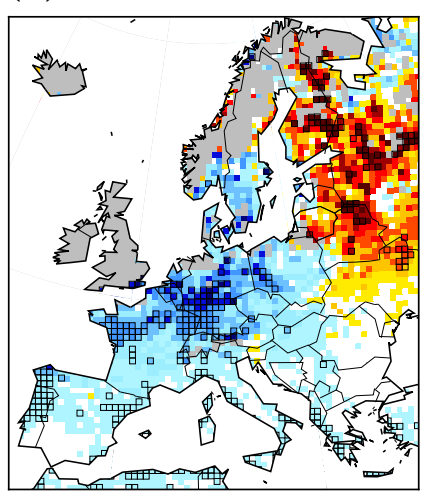

(e) ARI $\mathrm{AOD}_{c s}$

$s_{-}$corr with $(\mathrm{b})=-0.04$

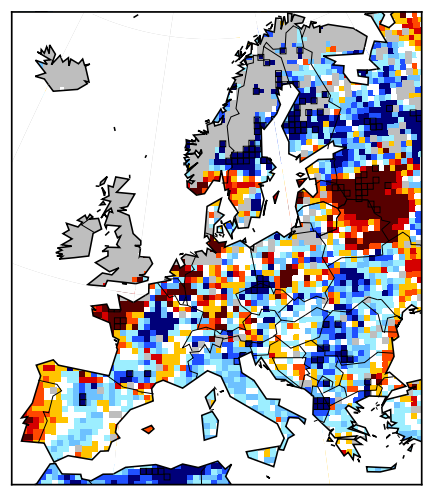

(c) ARCI RSDS

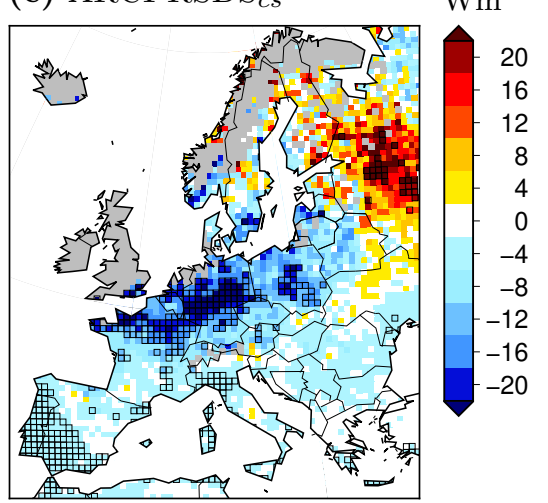

(f) ARCI $\mathrm{AOD}_{c s}$

$s_{-}$corr with $(\mathrm{c})=-0.18$

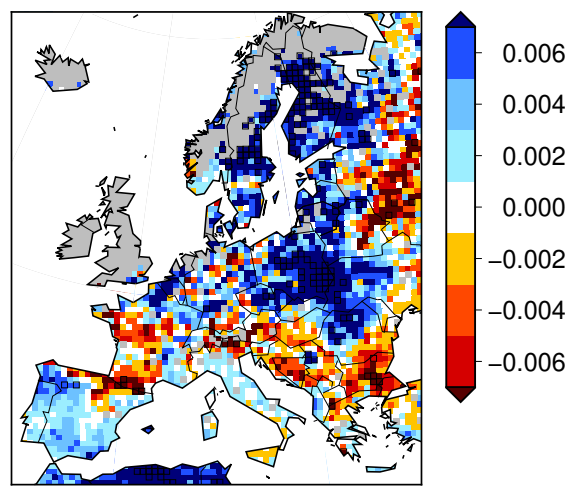

Supplementary Figure S13. As Figure 8 of the main manuscript but with plain differences depicted (non-relative values). Units: $\operatorname{RSDS}_{c s}$ in $\mathrm{Wm}^{-2}$, $\mathrm{AOD}_{c s}$ dimensionless. 
RSDS, CCT \& AOD DJF climatologies for 1991-2010: differences between experiments

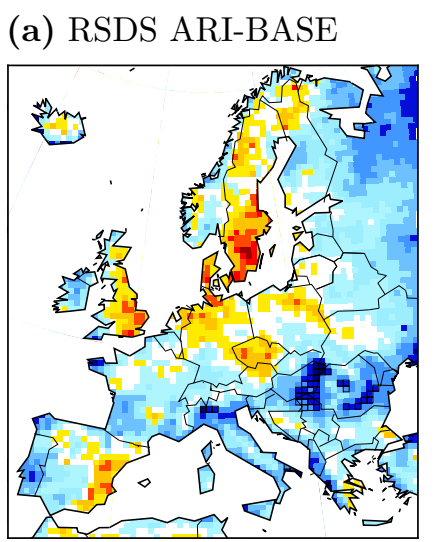

(d) CCT ARI-BASE

s_corr with $(\mathrm{a})=-0.35$

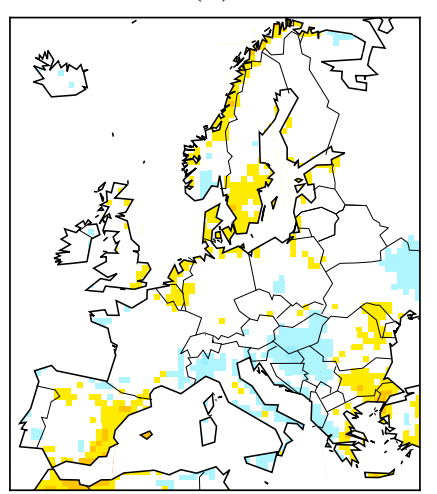

(g) AOD ARI

$s_{-}$corr with $(\mathrm{a})=-0.55$

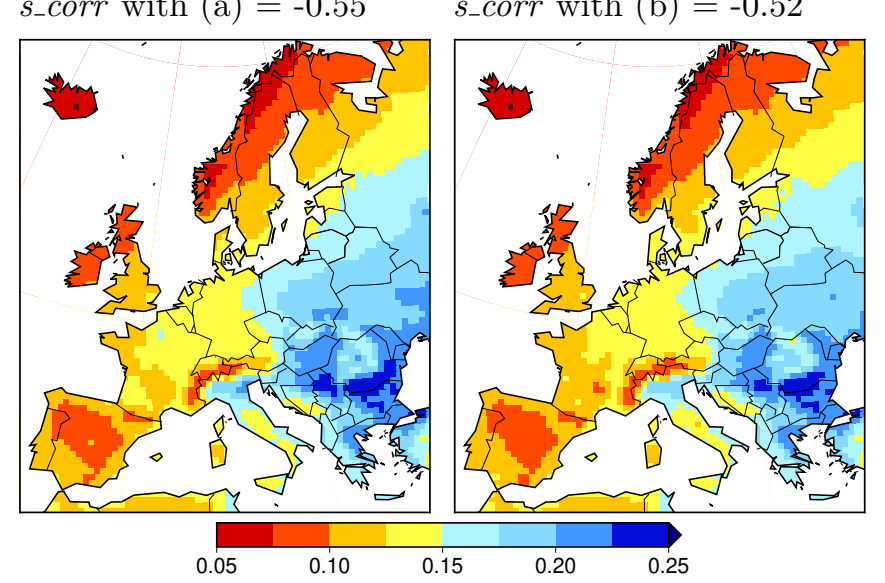

(c) RSDS ARCI-ARI

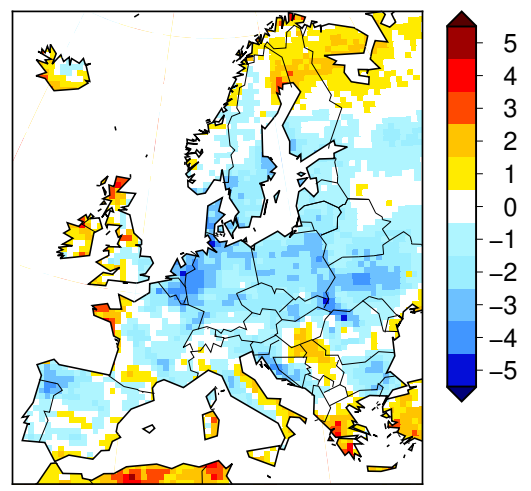

(f) CCT ARCI-ARI

$s_{-}$corr with $(\mathrm{c})=-0.53$

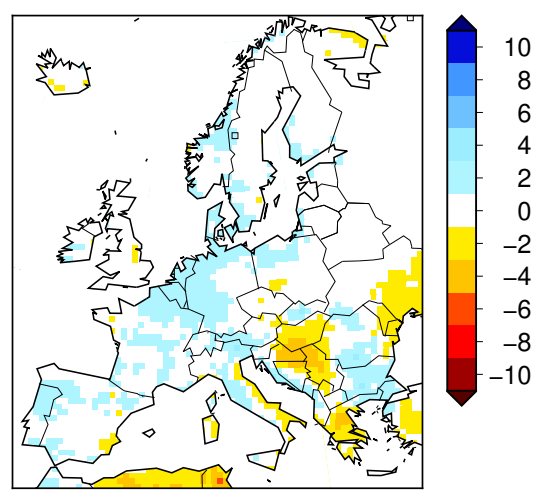

(i) AOD ARCI-ARI

$s_{-} \operatorname{corr}$ with $(\mathrm{c})=-0.09$

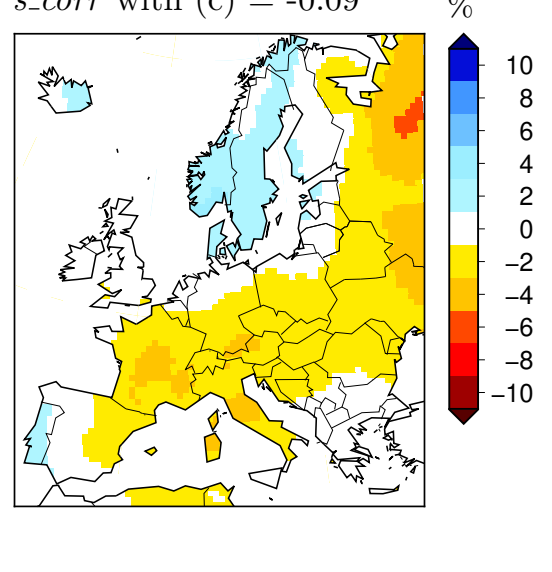

Supplementary Figure S14. As Figure 1 of the main manuscript but for the winter (DJF) season. 
$\mathrm{RSDS}_{c s} \& \mathrm{AOD}_{c s}$ DJF climatologies for 1991-2010: differences between experiments

\section{(a) $\operatorname{RSDS}_{c s}$ ARI-BASE}

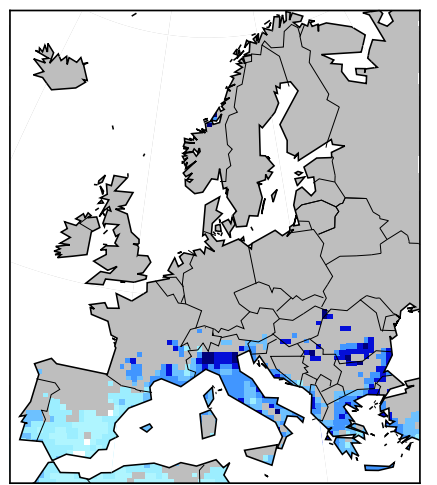

(d) $\mathrm{AOD}_{c s} \mathrm{ARI}$

$s_{-}$corr with $(\mathrm{a})=-0.91$

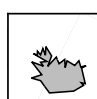

zing

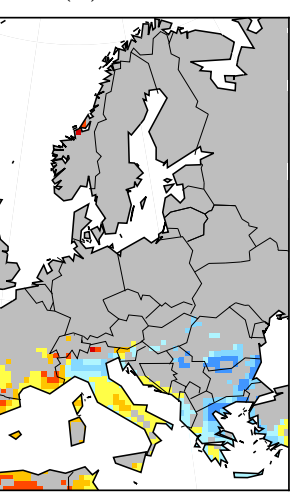

(b) $\mathrm{RSDS}_{c s}$ ARCI-BASE

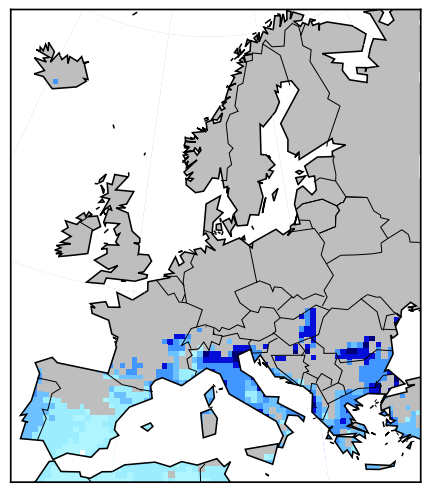

(e) $\mathrm{AOD}_{c s} \mathrm{ARCI}$

$s_{-}$corr with $(\mathrm{b})=-0.89$

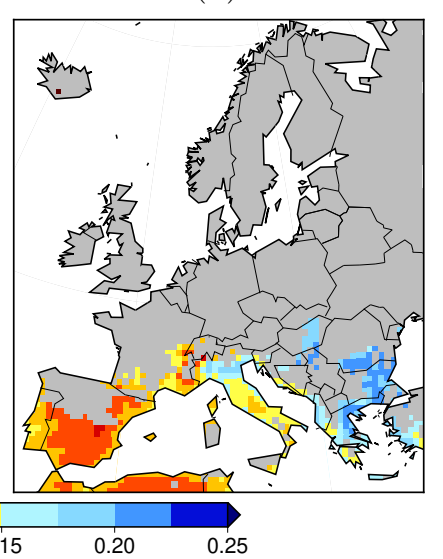

(c) $\operatorname{RSDS}_{c s}$ ARCI-ARI

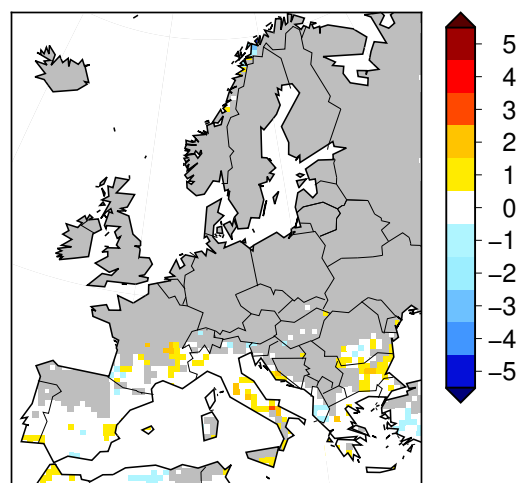

(f) $\mathrm{AOD}_{c s}$ ARCI-ARI $s_{-}$corr with $(\mathrm{c})=-0.51$

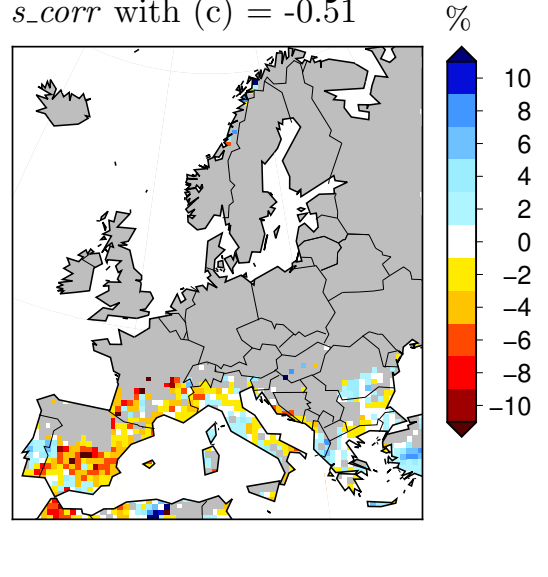

Supplementary Figure S15. As Figure 6 of the main manuscript but for the winter (DJF) season. 
RSDS, CCT \& AOD DJF changes (2031-2050 vs. 1991-2010)

(a) GCM RSDS

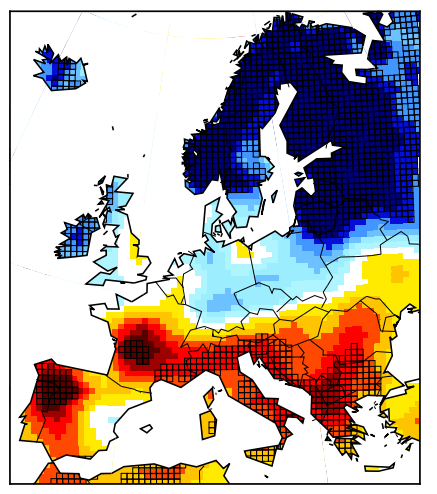

(e) GCM CCT

s_corr with $(\mathrm{a})=-0.88$

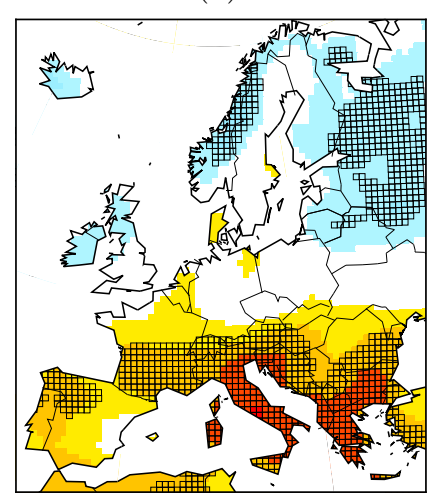

(i) GCM AOD

$s_{-}$corr with $(\mathrm{a})=0.03$

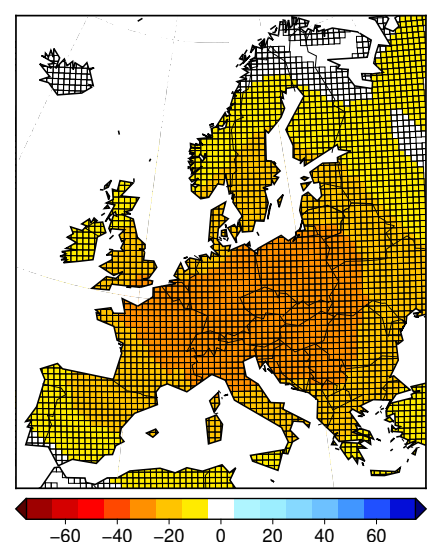

(b) BASE RSDS

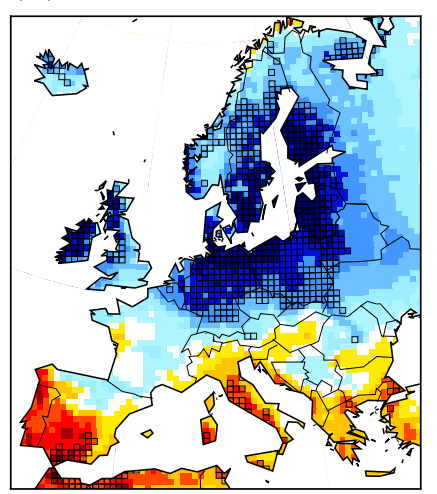

(f) BASE CCT

$s_{-}$corr with $(\mathrm{b})=-0.80$

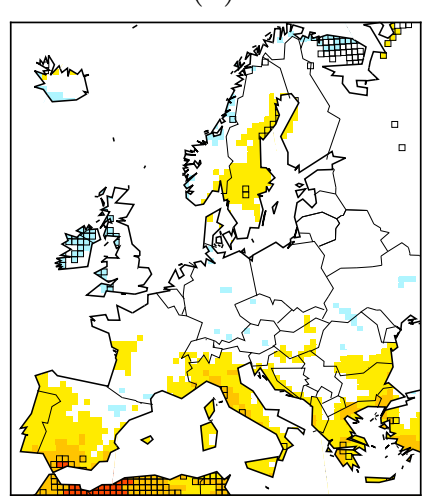

(j) BASE AOD

$s_{-}$corr with $(\mathrm{b})=$ N.A.

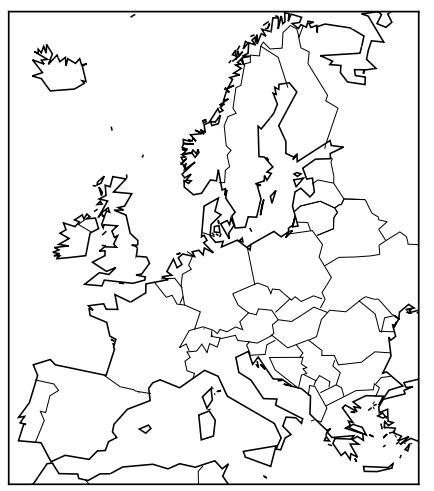

(c) ARI RSDS

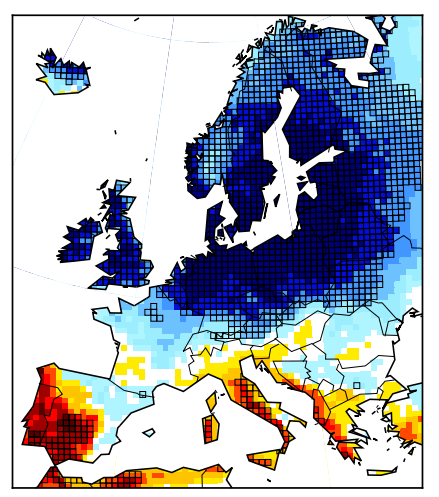

(g) ARI CCT

$s_{-}$corr with $(\mathrm{c})=-0.79$

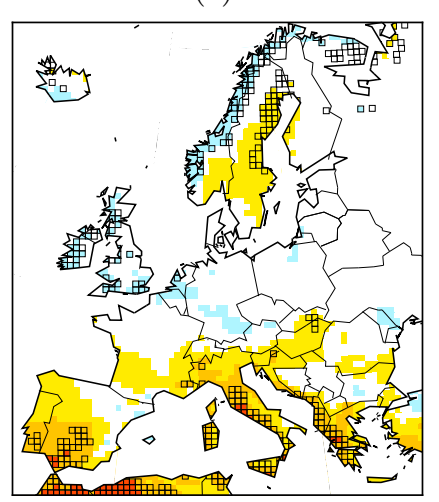

(k) ARI AOD

$s_{-}$corr with $(\mathrm{c})=0.37$

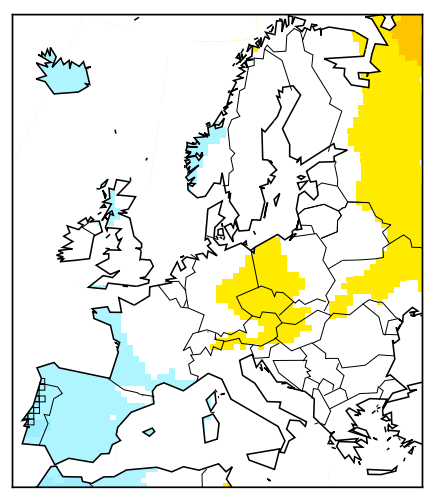

(d) ARCI RSDS

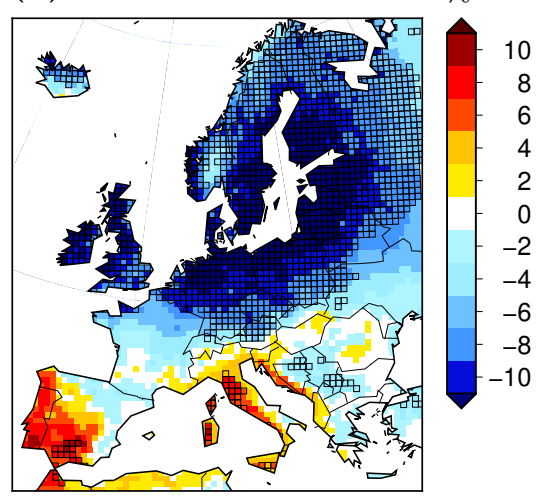

(h) ARCI CCT

$s_{-} \operatorname{corr}$ with $(\mathrm{d})=-0.67 \quad \%$

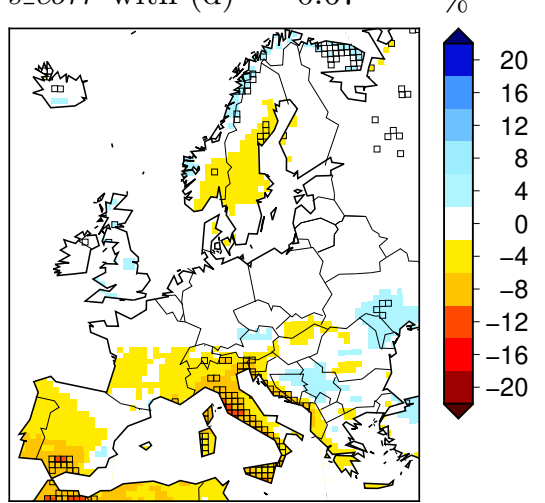

(1) ARCI AOD

$s_{-} \operatorname{corr}$ with $(\mathrm{d})=0.33$

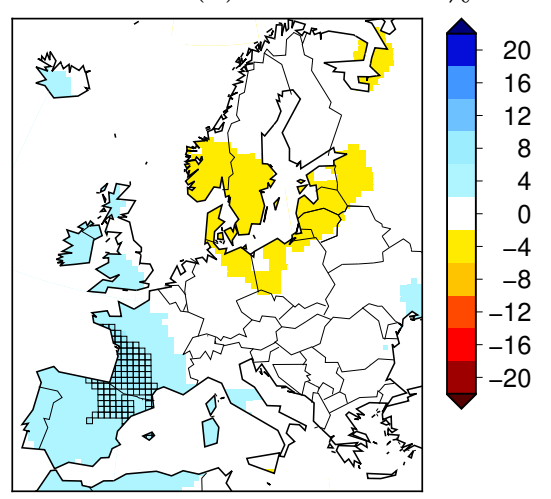

Supplementary Figure S16. As Figure 7 of the main manuscript but for the winter (DJF) season. 
$\operatorname{RSDS}_{c s} \& \mathrm{AOD}_{c s}$ DJF changes (2031-2050 vs. 1991-2010)

(a) BASE RSDS $c s$

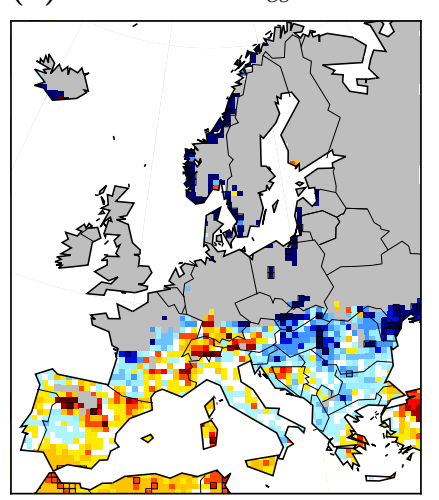

(d) BASE $\mathrm{AOD}_{c s}$

$s_{-} \operatorname{corr}$ with $(\mathrm{a})=$ N.A.

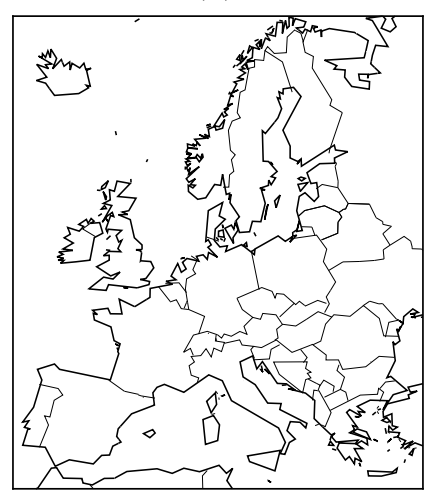

(e) ARI $\mathrm{AOD}_{c s}$

$s_{-}$corr with $(\mathrm{b})=0.28$
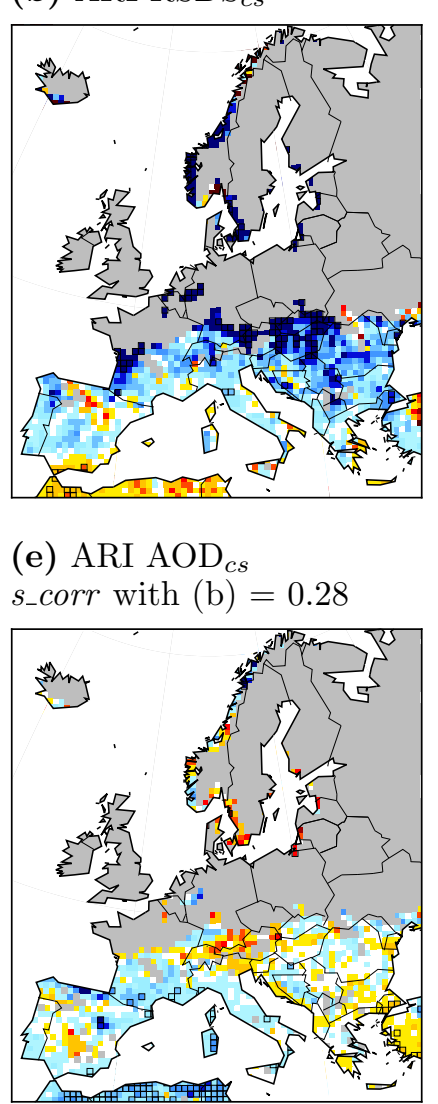

(c) ARCI RSDS Rs

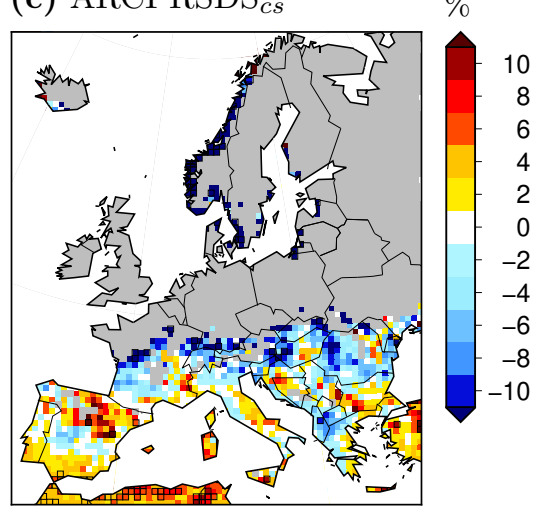

(f) ARCI $\mathrm{AOD}_{c s}$

$s_{-}$corr with $(\mathrm{c})=0.18$

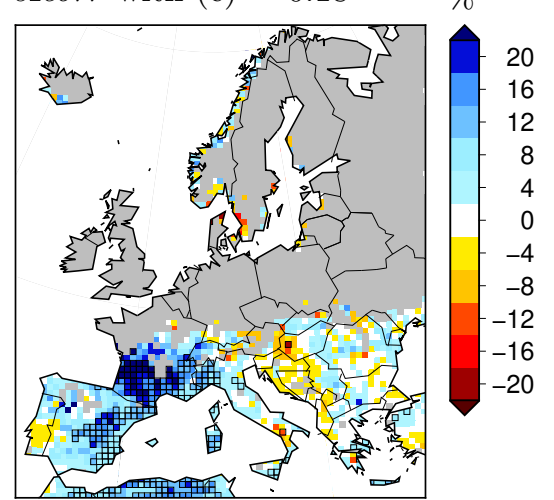

Supplementary Figure S17. As Figure 8 of the main manuscript but for the winter (DJF) season. 